\title{
Occupational Commitment From a Life Span Perspective:
}

\section{An Integrative Review and a Research Outlook}

\author{
Daniel Spurk ${ }^{1,2}$ \\ Annabelle Hofer ${ }^{2}$ \\ Anne Burmeister ${ }^{3}$ \\ Julia Muehlhausen ${ }^{2}$ \\ Judith Volmer ${ }^{4}$
}

${ }^{1}$ corresponding author

${ }^{2}$ Department of Work and Organizational Psychology, University of Bern, Switzerland

${ }^{3}$ Department of Organisation and Personnel Management, Rotterdam School of Management (RSM),

Erasmus University Rotterdam, the Netherlands

${ }^{4}$ Department of Work and Organizational Psychology, University of Bamberg, Germany

Correspondence address:

Daniel Spurk, $\mathrm{PhD}$

University of Bern

Department of Work and Organizational Psychology

Fabrikstrasse 8

3012 Bern, Switzerland

Email: daniel.spurk@psy.unibe.ch

Phone: +41(0)31-631-5386

Fax: $\quad+41(0) 31-631-8212$

\section{To cite this document:}

Spurk, D., Hofer, A., Burmeister, A., Muehlhausen, J., \& Volmer, J. (accepted). Occupational Commitment From a Life Span Perspective: An Integrative Review and a Research Outlook. Career Development International. 


\begin{abstract}
Purpose - The purpose of this review was to integrate and organize past research findings on affective, normative, and continuance occupational commitment (OC) within an integrative framework based on central life span concepts.
\end{abstract}

Design/methodology/approach - We identified and systematically analyzed 125 empirical articles (including 138 cases) that examined OC with a content valid measure to the here applied definition of OC. These articles provided information on the relationship between OC and four distinct life span concepts: chronological age, career stages, occupational and other life events, and occupational and other life roles. Furthermore, developmental characteristics of OC in terms of construct stability and malleability were reviewed.

Findings - The reviewed literature allowed us to draw conclusions about the mentioned life span concepts as antecedents and outcomes of OC. For example, age and tenure is more strongly positively related to continuance $\mathrm{OC}$ than to affective and normative $\mathrm{OC}$, nonlinear and moderating influences seem to be relevant in the case of the latter OC types. We describe several other findings within the results sections.

Originality/value - OC represents a developmental construct that is influenced by employees' workand life-related progress, associated roles, as well as opportunities and demands over their career. Analyzing OC from such a life span perspective provides a new angle on the research topic, explaining inconsistencies in past research and giving recommendation for future studies in terms of dynamic career developmental thinking. 


\section{Occupational Commitment From a Life Span Perspective:}

\section{An Integrative Review and a Research Outlook}

Occupational commitment (OC), which is broadly defined as the psychological link between a person and her or his occupation (Lee et al., 2000), represents a core concept for understanding an individual's career development and associated attitudes, behaviors, and choices (Blau, 1989, CooperHakim and Viswesvaran, 2005). Past research has shown that OC is related to, for instance, turnover intentions (Blau, 1989), job performance (Lee et al., 2000), and other commitment foci, such as work commitment (Cooper-Hakim and Viswesvaran, 2005), career satisfaction (Aryee et al., 1994), and work-nonwork issues (Okurame, 2012). Hence, OC affects an individual's work and life-related development, and can be a guiding mechanism for meaningful, identity-building career and life planning (Meyer et al., 2008).

Drawing upon a developmental perspective, some studies have analyzed OC or related constructs from a life span perspective, for instance, by using the concept of career stages (Aryee et al., 1994, Ng and Feldman, 2007), or by occasionally analyzing OC developments (Vandenberg and Scarpello, 1994). Taking a life span perspective involves focusing on the ways that different workand nonwork-related roles, opportunities, and demands — which are experienced throughout one's entire (working) life span—influence, and are influenced by, OC. Such a life span developmental perspective on OC is relevant and timely because fundamental changes in the workplace, such as increasingly aging workforces or ongoing labor market (de)regulations, have shaped an environment with high demands for organizational and occupational mobility and lifelong learning (Hall, 2002, Ng and Feldman, 2007, Zacher, 2015), where OC plays a central role. Moreover, individual role constellations and expectations (from oneself and others) within one's occupational and private life domain have become more individualized, dynamic, and complex (Carstensen, 2006, Greenhaus and Kossek, 2014), showing the relevance of applying a more dynamic life span perspective on OC development, as well as on its predictors and outcomes.

However, we are missing a systematic review that integrates research on OC from a life span perspective, and thus have yet to understand how OC influences, and is influenced by, changing 
patterns of roles, opportunities, and demands throughout one's life span. Instead, past meta-analytic work on OC has focused on conceptual issues, such as how different dimensions of OC are related to other work commitment foci (Cooper-Hakim and Viswesvaran, 2005). Consequently, life spanrelevant correlates (e.g., chronological age, career stages, and life events) were not analyzed. Another meta-analysis (Lee et al., 2000) analyzed only affective OC in relation to sociodemographic and work-related variables. Although some of the investigated correlates can be linked to a life span perspective (e.g., chronological age, tenure, or turnover), the meta-analysis did not integrate the results from the lens of a life span perspective. Moreover, since the meta-analysis (Lee et al., 2000) was conducted 19 years ago, recent research on OC has not been considered. In sum, past metaanalytical review work applied either a conceptual or more static perspective to OC, and did not aim to understand OC from a life span perspective.

Against this background, the aims of the current review were threefold. First, we provide a comprehensive overview of scholarly research on OC from a developmental life span perspective. We draw upon knowledge provided within theoretical and empirical work, and apply a systematic procedure for reporting central characteristics of relevant, published quantitative empirical studies (e.g., applied measurement of OC, study design, and study sample). Specifically, we integrate research results within an organizing framework based on theoretical approaches from life span (career) development (e.g., Lent and Brown, 2013, Baltes et al., 2014, Super et al., 1990). This framework focuses on how central life span concepts (i.e., chronological age, career stages, occupational and other life events, and occupational and other life roles) can be seen as drivers or outcomes (or both) of OC. Second, because a developmental perspective on OC optimally investigates effects on and of OC changes, we examine past longitudinal quantitative studies (e.g., Vandenberg and Scarpello, 1994) with regard to absolute mean changes and relative rank-order change in OC over time. Third, we develop a state-of-the-art agenda for future research on life spanrelated OC research, considering both theoretical and methodological aspects. Such a future research agenda focusing on developmental aspects of OC is specifically important for OC research because it 
aims to foster more dynamic research on OC, considering both life span-related concepts and the passage of time.

\section{The Concept of Occupational Commitment}

We conceptualize OC as a psychological link between a person and her or his occupation (Lee et al., 2000). Thus, someone with higher occupational commitment strongly identifies with and has positive feelings about their occupation (Blau, 1985). Although such an affective OC dimension described as a person's emotional attachment to their occupation (Blau, 2003) or as desire to remain in the occupation (Meyer et al., 1993) was predominant in past OC research, other important dimensions of $\mathrm{OC}$ do exist: normative $\mathrm{OC}$ is defined as an obligation to remain in the occupation, whereas continuance $\mathrm{OC}$ can be seen as a perceived cost associated with leaving the occupation (Meyer et al., 1993). Frequently applied measures for either one or all three OC dimensions include the OC scales from the Blau (2003), or modified versions of organizational commitment measures (e.g., Meyer et al., 1993). Usually, among the three OC dimensions, studies have shown the strongest, but moderately positive relationship between affective and normative OC, followed by a weaker, positive relationship between normative and continuance OC (Meyer et al., 1993). Affective and continuance OC are occasionally negative, or not related (Meyer et al., 1993), although the latest meta-analysis on this topic shows, on average, a positive correlation, with a high variability between studies (Cooper-Hakim and Viswesvaran, 2005). This overall picture points toward the importance of differentiating between the three OC dimensions within this review.

Past research used the terms occupation, profession, and career somewhat interchangeably in the commitment literature. We avoided using the term career commitment (Carson and Bedeian, 1994, Blau, 1985) because of ambiguity in the meaning of the term career. Career can be defined as a desired or planned pattern of work from career entry to retirement, or as involvement in a particular job, organization, or occupation (Meyer et al., 1993). Because we were interested in reviewing commitment as a psychological link between a person and her or his occupation (in the sense of conducting a particular line of work), we felt that the term occupation was more appropriate than the term career. Moreover, the Carson and Bedeian's (1994) Career Commitment Measure includes 
dimensions that are different form the meaning of OC understood here: career identity, career planning, and career resilience. We chose to use the term occupation rather than profession (Adler and Aranya, 1984) because both professionals and nonprofessionals can experience commitment to the work they do. Hence, in our opinion, occupation is more inclusive than profession, and therefore more in line with the conceptualization of commitment targeted here.

\section{A Life Span Perspective of Occupational Commitment}

Life span theories (e.g., Baltes, 1987, Baltes et al., 2014, Carstensen, 2006) provide a useful theoretical lens for understanding OC because they acknowledge the construct's developmental component. Accordingly, OC and an individual's work and life-related progress are interrelated, such that career and life planning affects OC. Its developments, in turn, can guide career and personal life decisions (Baruch et al., 2014, Klein et al., 2012, Ok and Vandenberghe, 2016). The general premise of life span development theories is that individuals face different challenges (e.g., becoming a parent or changing a job) across their life span, which require adaptation and decisions about changing resources, emphasizing individual development as a central aspect of human life (Lerner and BuschRossnagel, 2013). Specifically, life span approaches highlight the relevance of different interrelated concepts — which we refer to as life span concepts_-for human development and resource adjustment throughout one's life (e.g., Baltes et al., 2014, Carstensen, 2006, Super et al., 1990): chronological age, career stages, occupational and other life events, and occupational and other life roles. We argue that these interrelated life span concepts are also useful for organizing and integrating research on OC as a psychological link between a person and her or his occupation. The organizing framework for this review is depicted in Figure 1.

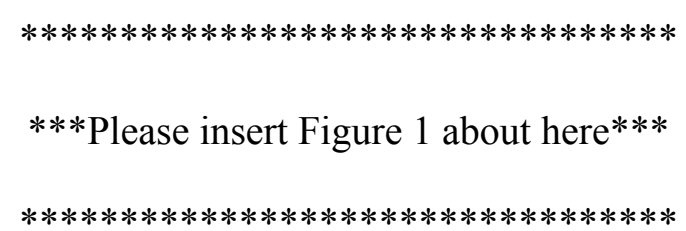

The organizing framework displays the links of chronological age [Research Question (1)], career stages [Research Question (2)], occupational and other life events [Research Question (3)], and occupational and other life roles [Research Question (4)] with OC, as well as the often assumed 
interrelationships between the four different life span concepts (e.g., Baltes et al., 2014; Super, 1990; Carstensen, 2006). In addition, the organizing framework highlights the possibility of occupational and other life events as outcomes of OC [Research Question (5)], and the potential dynamic nature of OC, which accounts for feedback loops on OC changes [Research Question (6)].

\section{Literature Search and Selection}

We conducted a web-based search to identify studies that examined OC within the fields of psychology, social sciences, and business/management. First, we searched the Web of Science for the terms "occupational commitment," "career commitment," "professional commitment," and "vocational commitment" within the search fields title and abstract (for a similar strategy see also Lee et al., 2000). We did not restrict our search by publication date, but focused on publications in English. Second, so as not to miss central studies, we also searched central journals in these fields using Scopus (e.g., Journal of Applied Psychology, Journal of Management, and Journal of Vocational Behavior). We included only articles in our review that operationalized OC with a content-valid measure, according to the definition of OC applied here, and hence, either assessed as affective, normative, or continuance commitment (or a combination of these). For example, articles which studied career salience (Mihelič, 2014), career involvement (Gould, 1979), other commitment foci (Brooks et al., 1995), commitment to career choices (Blustein et al., 1989), or career planning, career identity, and career resilience (Carson and Bedeian, 1994), were excluded. A full list of measures that were included in the review can be seen in Appendix 1.

We focused on articles and results that are especially useful for understanding OC from a life span perspective. For example, we inspected the type of analyzed correlates (e.g., antecedents and outcomes of OC), and rated them according to the life span concepts introduced above that represented the organizing framework of the review (e.g., an article that analyzed marriage as an antecedent was relevant because married individuals hold the role of spouse, and such articles are relevant for Research Question 4 in Figure 1; or a study that analyzed retirement intentions as an outcome was relevant because these intentions represent a proxy for occupational events in late career stages, see Research Question 5 in Figure 1). Furthermore, every article that measured OC across 
several time points was included because it can potentially inform us about dynamic OC developments over a person's career (see number 6 in Figure 1). After this screening process, 125 articles were identified as relevant for this review. Based on these articles, we identified 138 relevant cases; for example, some articles included more studies (e.g., Otto et al., 2010) or more (sub)samples in one article (e.g., Burke et al., 2015).

Furthermore, we coded a number of aspects that are directly related to our review goals or help summarize the characteristics of the identified studies, and derive recommendations for future research (e.g., applied measurement of OC, study design, and study sample). In the whole selection and coding process, at least two members of the author team rated the same article or variable. In case of nonconformity, the decision was discussed until full agreement in the author team was found. In Table 1, we summarize frequencies and proportions of different study characteristics. In the following sections on the different life span concepts related to different research questions (see Figure 1), we first provide a theoretical rational for the derived research question, directly followed by a results section reviewing empirical studies relevant to answering the respective research question.

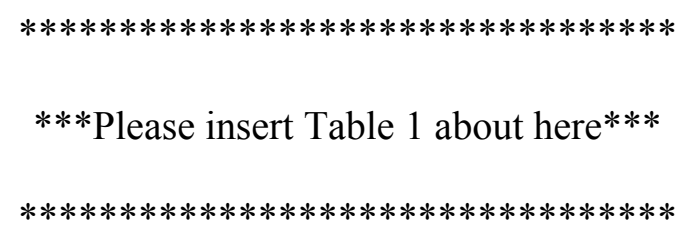

\section{Chronological Age as an Antecedent of Occupational Commitment}

Chronological age refers to an individual's calendar age (Kooij et al., 2008), that is to say, the time that has passed since an individual's birth. For reasons of simplicity, when we refer to age in the following review, it is in the sense of chronological age. Authors in OC research have proposed that age should correlate positively with OC (Reilly and Orsak, 1991), specifically continuance OC, because of accumulating investments during a career in an occupation (De Vos et al., 2017), and presumably reduced career options for older employees due to limited alternatives (Tempest and Coupland, 2017). This argument builds on side-bet theory (Becker, 1960), which refers to an accumulation of investments that would be lost if the individual leaves the occupation. The theory 
reasons that side-bets tend to increase over one's life span, as individuals' investments aggregate over time. Therefore, age should be positively related with continuance OC.

Psychological life span theories - specifically socioemotional selectivity theory (Carstensen, 2006) - approach the same topic from another perspective, which might also help to explain how and why age is associated with OC. However, in contrast to side-bet theory, socioemotional selectivity theory might also explain the relationship of age and affective and normative OC. Socioemotional selectivity theory focuses on individual experience and attitudinal and behavioral consequences of aging in terms of changing goal priorities (Hertel and Zacher, in press). Accordingly, based on an individual perception of one's future time remaining, individuals identify and prioritize different values and goals. Younger individuals who perceive their remaining time as unlimited tend to focus on goals that can be realized in the future, such as accumulating knowledge and advancing their personal network. By contrast, older individuals who perceive their time as limited focus on the present and prioritize social and emotional goals that contribute to their current well-being (Carstensen et al., 1999, Carstensen, 2006, Erikson, 1963).

For example, if workers perceive their future time as limited starting in mid-to-late adulthood, they tend to focus on emotional goals and opportunities for generativity, rather than advancing their own career (Henry et al., 2015, Henry et al., 2017, Wang et al., 2015). Following, work and life roles and related values are also affected by age (Kooij et al., 2008), which, in turn, affect the psychological link between a person and that person's occupation (Van Vuuren et al., 2007). A prioritization of different values and goals during the aging process might affect a person's emotional attachment to their occupation because of changing priorities across life domains, and hence, nonwork domains might become more important, at least for a certain period of time. Because such role transitions are becoming more frequent (Mainiero and Sullivan, 2005), age might show nonlinear relationships with affective OC. Finally, an accumulation of occupational events and new roles associated with the aging process should put the person within their current occupation into a situation with multiple reciprocity bonds (Borgatti and Foster, 2003), which should positively affect a 
person's normative OC. However, due to the dynamic nature of these bonds, the relationship might not necessarily be linear.

In sum, when referring to life span theories, socioemotional selectivity theory specifically draws a complex picture of the relationship between age and affective and normative OC, even assuming the possibility of nonlinear and negative relationships with age. Hence, we expect the relationship between age and affective and normative OC to be relatively complex, whereas the linear and positive assumption from side-bet theory is only valid for continuance OC.

Research Question 1: Is age linearly and positively related to affective, normative, and continuance OC, or is the relationship more complex, and expressed in a diverse correlation pattern?

\section{Results: Correlation Pattern Between Age and Occupational Commitment}

We identified 70 cases that used age as a continuous or categorical (i.e., in terms of age groups) antecedent of OC, mostly considering affective (i.e., 70 correlations), but also normative (i.e., 17 correlations) and continuance (i.e., 13 correlations) commitment. Regarding affective OC, the results of the analyzed studies suggest that, on average, the relationship between age and OC tends to be small and positive (average $r=.07$ ). However, results were highly heterogeneous and ranged from negatively significant, to nonsignificant, and positively significant (minimum $r=-.22$, maximum $r=.71$ ). This pattern for affective OC corresponds to existing meta-analytical evidence (Lee et al., $2000)$, which has pointed to a small positive $(r=.07$, based on a $k$ of 26$)$, and highly heterogeneous age-affective OC relationship (i.e., only $19.25 \%$ of the variance between effect sizes in the metaanalysis were accounted for by artifacts).

Regarding normative commitment, the correlation pattern was very similar (average $r=.05$, minimum $r=-.11$, maximum $r=.37$ ); however, for continuance commitment, the average correlation seemed to be slightly more positive. Additionally, although the smallest correlation was slightly negative, it was nonsignificant (average $r=.15$, minimum $r=-.05$, maximum $r=.36$ ). These findings suggest that the correlation pattern is more diverse for age and affective and normative OC, compared to age and continuance OC. Moreover, because all significant correlations were positive for age and 
continuance OC, assumptions from side-bet theory (i.e., linear positive relationship) seem to be valid only for continuance OC, but not for the other types of OC.

To provide some concrete examples, Blythe et al. (2008) examined nurses of four different age groups (20-29, 30-29, 40-49, and 50+), and found that the youngest age group was more emotionally attached to their occupation compared to the other three older age groups. In addition, Christensen and Woodland (2016) reported lower affective OC for older and non-traditional students compared to traditional students who were younger than 25 years old. These findings, suggesting that age and OC might occasionally be negatively related, can be explained by borrowing from psychological life span theories, and more specifically, socioemotional selectivity theory (Carstensen et al., 1999). Accordingly, younger individuals are expected to be more committed to their occupation compared to older individuals because they tend to focus on goals that can be realized in the future, based on their unlimited time perspective. Moreover, a substantial amount of studies have reported nonsignificant relationships between age and affective (Zhou et al., 2015) and normative OC (Irving et al., 1997). Additionally, studies that obtained positively significant correlations (e.g., Cherniss, 1991, Morrow and Wirth, 1989, Tang et al., 2012) reported highly heterogeneous effect sizes. For example, Morrow and Wirth (1989) reported a correlation of $r=.14$ for age and affective OC, whereas Cherniss (1991) identified a correlation of $r=.71$ between age and affective OC.

In sum, at least for affective and normative OC, the heterogeneity of results suggests that the relationship between age and OC might be nonlinear, or that moderators might influence the direction and the strength of the relationship. Unfortunately, existing evidence on possible nonlinearity and moderators of the link between age and OC is very limited.

\section{Results: Nonlinear Relationships and Moderating Influences}

Regarding nonlinear relationships, one article that operationalized age as a dichotomous variable $(1=>40$ years $)$ found no quadratic effects of age on affective OC within a sample of academic and support staff from Dutch universities (Yousaf et al., 2015). However, due to the dichotomization of the age variable, this analysis suffered from an artificial reduction in variance. Two articles examined moderators of the link between age and OC. The first article found that culture 
(China vs. Russia) had a moderating influence such that age was positively related with all three types of OC within a Chinese sample, and negatively related within a Russian sample (Burke et al., 2015). The second article expected that older women (vs. men) are more committed, but did not find that gender moderated the link between age and affective OC in a sample of MBA students (Bishop and Solomon, 1989).

\section{Summary}

Regarding Research Question 1, the results of our review suggest that the relationship of age and OC differs by OC type. The correlation pattern between age and affective and normative OC is highly diverse across studies, indicating a relatively complex pattern. However, due to studies that explicitly assumed, or at least provided data about, nonlinear or moderated age-OC relationships, it might be too early to establish reasons for this complex correlation pattern. By contrast, although individual studies identified nonsignificant relationships between age and continuance OC, the correlation pattern is relatively homogeneous, and, on average, the relationship is positive. Finally, one main methodological shortcoming of the reviewed studies was that several studies used age as a categorical variable in terms of artificially defined age groups (even via median splits) rather than using age as a continuous variable, thereby unnecessarily reducing the variables' variance and the likelihood of detecting significant effects.

\section{Career Stage as an Antecedent of Occupational Commitment}

Different models of career stages exist, but Super's theory of career development can be regarded as the most prominent (Low et al., 2016). Super's (1953) theory proposes that career development is a lifelong process, and that individuals progress through four career stages in their life span, which are related to active involvement in the world of work: (1) exploration, (2) establishment, (3) maintenance, and (4) disengagement. Individuals in the exploration stage are discovering their interests and are entering into a new occupation. Individuals in the establishment stage usually increase their commitments to their occupation, aim to establish their competence, and focus on advancement and growth. Individuals in the maintenance stage concentrate on maintaining their selfconcept and the status quo, whereas individuals in the disengagement stage aim to develop a self- 
concept that is independent of their occupational life, whereas retirement issues become more salient. Against this background, career stages are frequently operationalized either as age or as tenure (Bishop and Solomon, 1989, Adams, 1999), whereas against the possibility of several types of career mobility, tenure is a closer proxy for career stages than age (Mount, 1984, Stumpf and Rabinowitz, 1981). Furthermore, new career models (Hall et al., 2018, Sullivan and Baruch, 2009, Arthur et al., 2005) highlight that individuals might recycle through different career stages, for example, when they change jobs or occupations later in life, or experience more frequent micro-stages, such as learning cycles within one career stage.

In sum, similarly to assumptions related to age, these arguments suggest that $\mathrm{OC}$ might change according to one's career stage because passing through career stages is related to experiencing specific occupational events and facing changing roles and tasks, which, again, are related to individual values and goals. Moreover, more frequent changes and re-experiences of career stages requires continuous adjustment of resources. These ongoing adaptations should affect an individual's sense of emotional attachment to their occupation, obligation to remain in the occupation, and perceived costs associated with leaving the occupation. From a side-bet perspective, again, continuing to late career stages in one and the same occupation without re-experiencing former stages or micro-cycles should be linear and positively related to continuance OC. However, from a more dynamic life span perspective, the relationship between career stages and affective and normative OC seems to be more complex, and might be nonlinear and/or be explained by a third variable, such as changing role expectations, value changes, or resource adjustments.

Research Question 2: Is the passage through career stages (i.e., tenure) linearly and positively related to affective, normative, and continuance OC, or is the relationship more complex, and expressed in a diverse correlation pattern?

\section{Results: Correlation Pattern Between Tenure and Occupational Commitment}

We identified 50 cases that used occupational tenure as a continuous or categorical (i.e., in terms of tenure groups) antecedent of OC, mostly considering affective (i.e., 50 correlations), but also normative (i.e., 15 correlations) and continuance (i.e., 12 correlations) commitment. Regarding 
affective OC, the results of the analyzed studies suggest that, on average, the relationship between occupational tenure and OC tended to be small and positive (average $r=.04$ ). However, similar to age reported above, results were heterogeneous and ranged from negatively significant, and nonsignificant, to positively significant (minimum $r=-.27$, maximum $r=.32$ ). This correlation pattern corresponds to existing meta-analytical evidence (Lee et al., 2000), which has pointed to a small, positive, and heterogeneous occupational tenure-affective OC $(r=.09$, based on a $k$ of 11 , $24.99 \%$ of the variance between effect sizes accounted for by artifact). Regarding normative OC, the correlation pattern with occupational tenure (average $r=02$, minimum $r=-.30$, maximum $r=.24$ ) was very similar to the one for affective OC; however, for continuance OC, the average correlation with occupational tenure seemed to be clearly positive, and there were no negative correlations (average $r=.21$, minimum $r=.01$, maximum $r=.40$ ).

We also identified some studies that provided a correlation between organizational or job tenure and OC; however, the number was smaller (15 for affective OC, three for normative OC, and only one for continuance OC). For affective OC, where we identified enough studies to allow some conclusions, the correlation pattern for organizational tenure was very similar to the pattern identified for occupational tenure.

For a better understanding of the underlying studies and the relatively heterogeneous effects for affective and normative OC, we briefly refer to some results in more detail. One of the largest significant, negative correlation between occupational tenure and affective OC $(r=-.21)$ was found within a sample of nurses from the United States. (Grover, 1992). The strongest negative and significant correlation between occupational tenure and normative OC $(r=-.30)$ was found in a sample of British and Chinese public and private industry accountants, whereas the correlation with affective OC was nonsignificant and, with continuance OC, was positive (Snape et al., 2008). The highest correlation between occupational tenure and affective OC $(r=.32)$ was observed in a study by Thompson and Van De Ven (2002) about organizational change and a sample of physicians, whereas the highest correlation between occupational tenure and normative OC $(r=.24)$ was found in a sample of registered nurses from the United States. (Meyer et al., 1993). Within this sample, all types 
of OC were positively related to occupational tenure. These results underline the rather complex pattern of relationships between tenure and affective and normative OC specifically, showing that even within the same population (i.e., nurses) from the same country (i.e., the United States.), the correlation between tenure and OC might lean toward the opposite direction. The underlying reasons for this diversity in effect sizes is not well understood, and only a limited number of studies have explored these complex patterns, focusing on nonlinearity and moderating factors.

\section{Results: Nonlinear Relationships and Moderating Influences}

While reviewing the literature, we identified six cases that investigated nonlinear or moderated relationships. Three cases examined nonlinear relationships. First, the article by Klassen and Chiu (2011) found quadratic effects of occupational tenure on different types of teaching selfefficacy. Teachers with a medium long tenure (20 to 25 years) showed the highest self-efficacy values in comparison with shorter or longer tenure. Because teaching self-efficacy was positively related to affective OC, the results suggest that occupational tenure can have an indirect and curvilinear relationship with affective OC via self-efficacy. Second, and providing even more support for nonlinear relationships, in another sample of teachers, quadratic effects of occupational tenure on affective OC were found (Mueller et al., 1999). Third, within a sample of R\&D staff of two Korean electronics companies, cubic developments of affective OC within the first 18 months of organizational tenure were observed, followed by a stabilization in later career stages (Chang and Choi, 2007). Taken together, these results suggest that —in line with assumptions of career stage theory and new career theories - the relationship between tenure and affective OC might be nonlinear.

Three articles examined possible moderators of the link between tenure and OC. First, Tang et al. (2012) showed that age group or generation moderated the link between tenure and normative OC, such that the link was nonsignificant for Generation X and positive for baby boomers. Second, organizational tenure was positively related with a composite score of all three types of OC in a Chinese sample, and negatively related in a Russian sample, suggesting that culture affects the direction of the link between tenure and OC (Burke et al., 2015). Third, gender affected the tenure- 
OC link (Cunningham et al., 2012), such that occupational tenure was positively related to all three types of OC for men, but only related to continuance OC for women.

\section{Summary}

In sum, regarding Research Question 2, in case of affective and normative OC, the relations between career stages and OC might be more complex compared to continuance OC, such that nonlinear relationships or moderating influences might be evident. Nonlinear effects have already been observed for affective OC, but have not been investigated for other types of OC. Moreover, in the studies reported here, the relationship of tenure and affective OC and normative OC varied by specific group memberships (i.e., gender, age, and country), whereas the relationship of tenure and continuance OC was not affected by these groups. Moreover, regarding continuance OC, the review findings of the correlational pattern suggest a positive relationship with the progress through classical career stages (in terms of tenure). It is worth mentioning that although a substantial amount of studies reported a correlation of (occupational) tenure and OC, not all of these studies have built their theoretical framework and hypotheses against the background of career stage-specific theories (for exceptions see Reilly and Orsak, 1991, Adler and Aranya, 1984). A further shortcoming of this research stream is that studies sometimes confound career stages with age groups, which cannot be seen as a valid indicator of career stages within the context of new careers.

\section{Occupational and Other Life Events as Antecedents of Occupational Commitment}

Occupational events (e.g., losing one's job, occupational restructuring) and other life events (e.g., marriage, death of loved one) can be defined as "change events, that is events that precipitate movement from one equilibrium or the steady state to another" (Holmes and Rahe, 1967b, p.216). Occupational and other life events are often framed as stressful events that necessitate individuals to readjust after the event happened (Holmes and Rahe, 1967a), but can also be framed as positive, for example, against the background of positive career shocks, such as getting an unexpected promotion (Akkermans et al., 2018). Events can be categorized in terms of their impact (i.e., desirable vs. undesirable) and their controllability (i.e., controllable vs. uncontrollable). For example, marriage 
would be — at least in Western cultures — a desirable and controllable event, whereas losing one's job might represent an undesirable and often uncontrollable event.

Occupational and other life events and OC are assumed to be associated with each other because such events affect an individual's cognitive and affective processing of internal and external states, and may also change the constellation and experience of the roles held by oneself and others. Therefore, OC should also be affected by such events (Klein et al., 2012). Furthermore, new career models (Hall et al., 2018, Sullivan and Baruch, 2009, Arthur et al., 2005) assume that individuals are more frequently confronted with potentially OC-changing events and shocks (e.g., job loss, job change, relocation of family home). Regarding the OC dimensions, events have the potential to affect one's own identity, can be related to diverse reciprocity bonds, and may also affect the estimation of occupation-related side-bets (Holmes and Rahe, 1967a, Wallace, 1997). Therefore, occupational and other life events might affect the emotional attachment to an individual's occupation, the obligation to remain in the occupation, and the perceived costs associated with leaving the occupation.

Research Question 3: Which occupational and other life events are related to affective, normative, and continuance OC?

\section{Results: Overview}

We identified only a few studies that explicitly focused on such discrete events and their effects on different kinds of OC. The majority of studies focused on occupational events, and sometimes subjective perceptions about happenings and the event per se was not operationalized. Overall, we identified three broader clusters of occupational events: (a) work-related transitions (e.g., job entry), (b) on-the-job events (e.g., loss of rank), and (c) macro-events (e.g., economic crisis).

\section{Results: Work-Related Transitions and Occupational Commitment}

Within a sample of early career-medical technologists, the pure event of changing once job was no related to affective OC up to one year later (Blau, 1999). Interestingly, another article showed that changing one occupation to another (e.g., in this sample, for instance, from nursing to law or from social worker to real estate salesperson) was associated with a drop in affective OC over time (Cherniss, 1991). The article by Thompson and Van De Ven (2002) has suggested that enabling 
characteristics of organizational change (e.g., being informed about changes, leader support of changes) are important for protecting affective OC from being impaired by role transitions (event: transition from self-employment to employment status). In a sample of hospitality college students, Wang et al. (2014) showed that internship anxiety before starting the internship was negatively related to affective OC. Hence, expectations (in terms of anxiety) about occupational events in terms of transitions (i.e., moving from student to intern role) might already be related to affective OC. Finally, within a sample of nurses who started their occupational training, affective OC declined throughout the duration of their training (Arnold, 1990).

\section{Results: On-the-Job Events and Occupational Commitment}

The article by Arnold (1990) showed that unpleasant job experiences during the first months of nurses training, such as ineffective teaching and instruction on the ward, negatively predicted change in affective OC. Moreover, anticipating the possibility of unpleasant job-related events (e.g., loss of rank, getting fired, getting pressured to accept a lower salary) was negatively related to OC within a sample of professors working in universities with post-tenure review policy (Ugboro and Obeng, 2015). Finally, one study analyzed whether being exposed to threatening physical and verbal events at work influenced affective OC of nurses (Zhou et al., 2015). Results showed that experiencing the event of verbal aggression is detrimental for affective OC, specifically under conditions of low interpersonal influence. Furthermore, having an injury at work was also negatively related to affective OC.

\section{Results: Macro-Events and Occupational Commitment}

The study by $\mathrm{Wu}$ et al. (2012) investigated the extent to which an outbreak of a severe acute respiratory syndrome (SARS) in Taiwan affected nurses' commitment to their occupation. The results showed that the SARS outbreak did not impact affective OC and the willingness to accept assigned jobs. On the contrary, affective OC was even higher during the outbreak (compared to two years before). These results suggest that external pressures on an occupation might even increase affective OC. Further, results from a study with Korean travel agency employees in times of a global economic crisis (Kim and Chang, 2014) suggest that affective OC might be an important resource for navigating 
the complexity of today's careers (Akkermans et al., 2018, Hall et al., 2018). Specifically, their results showed that affective OC buffered the detrimental effect of job insecurity on organizational turnover intentions. Some other studies framed their work against the background of macro-events, such as the economic reforms in Russia (Osinsky and Mueller, 2004) or China (Liu and Cohen, 2010), but did not investigate macro-events related influences in more detail.

\section{Summary}

In sum, regarding Research Question 3, existing studies have primarily focused on workrelated transitions, on-the-job events, and macro-events. Events not related to the work domain, such as having a child or the death of a loved one, have not been the focus of past research. Moreover, nearly all studies measured the affective OC component, yet failed to investigate separate effects on normative or continuance OC. The results regarding work-related transitions were rather mixed; however, the only study that investigated occupation changes showed a decrease in affective OC after an actual occupation change. Regarding on-the-job events, one conclusion might be that positively experienced or anticipated events are positively related to affective $\mathrm{OC}$, whereas negatively experienced or anticipated events are negatively related to affective OC. By contrast, negative macroevents did not negatively impact affective OC in the studies reviewed here. Finally, research in this area seems specifically restricted due to the fact that events have not been systematically theorized and classified, for instance, along the dimensions of impact (i.e., desirable vs. undesirable) or controllability (i.e., controllable vs. uncontrollable). Moreover, considering the definition of life events (Holmes and Rahe, 1967a), although a detailed investigation of events warrants a longitudinal examination (before and after the event), the majority of these studies applied cross-sectional designs.

\section{Occupational and Other Life Roles as Antecedents of Occupational Commitment}

Super (1980) introduced the concept of life roles within and outside of work to specify the variety of roles that people occupy during their career as they mature. He distinguished among nine major life roles that are likely to be occupied by most people at some point in their lives, which can also be enacted at the same time: (1) child, (2) student, (3) leisurite, (4) citizen, (5) worker, (6) spouse, (7) homemaker, (8) parent, and (9) pensioner. The order in which these roles are occupied 
(with exception of the role of child) might differ between persons, and the type, number, temporal importance, and emotional involvement associated with these roles is likely to vary with age and career stages due to the different developmental tasks individuals encounter. Following side-bet theory (Becker, 1960), individuals with higher extra-work responsibilities (e.g., as spouse and parent) should show higher levels of continuance OC because they do not want to lose their side-bet investments, due to the high levels of extra-work responsibilities and associated higher costs when leaving the profession (Howes and Goodman-Delahunty, 2014). Hence, occupying multiple roles or changing roles should affect side-bet evaluations and perceptions of continuance OC.

Moreover, when integrating assumptions from role theory (Biddle, 1986) and social identity theory (Tajfel, 1981) within a life span perspective, highly affective OC may result either from the increasing familiarity to the role individuals embody over the course of their careers (i.e., liking due to continued exposure), or from the desire for a role that might be attained by remaining in that occupation (e.g., aiming for the role of a leadership or expert position in the future). In the latter case, affective $\mathrm{OC}$ can be seen as a result of role expectations about upcoming life phases. Furthermore, role expectations especially from other individuals in the occupational (e.g., supervisor) or private (e.g., spouse) life domain might specifically affect perceptions of being obligated to remain in the occupation (i.e., normative OC). Finally, new career models, such as the kaleidoscope career also specifically highlight the relevance of integrating different roles within and across life domains (e.g., work/family) as potential correlates of OC (Mainiero and Sullivan, 2005). In sum, occupational and other life roles and OC seem to be interconnected, such that differences in the enactment of ongoing, expanding, and anticipated roles and group memberships can affect individuals' $\mathrm{OC}$ ( $\mathrm{Ng}$ and Feldman, 2007).

Research Question 4: How are actual, anticipated, or conflicting occupational and other life roles and expectations of others about these roles related to affective, normative, and continuance OC?

\section{Results: Overview}


We identified a number of articles that included variables related to life roles as antecedents of OC. These roles primarily referred to the life role as spouse (i.e., marriage) and the life role as parent (i.e., children at home, number of dependents). However, occupational roles (i.e., having a managerial position or being a professional) have also been investigated. Moreover, valuing the work or other life domains were one antecedent cluster for OC. Finally, interactions between work and other life roles (e.g., enhancement or conflict between the role as a parent and a worker) have been investigated relatively frequently.

\section{Results: Life Role as Spouse and Occupational Commitment}

Focusing on the life role as spouse, we identified 21 cases that provided information about marriage as an antecedent of OC, heavily focusing on affective (i.e., 21 correlations) and neglecting normative (i.e., two correlations) and continuance (i.e., one correlations) commitment. Regarding affective OC, the results of the analyzed studies suggest that, on average, the relationship between marriage and $\mathrm{OC}$ is about zero (average $r=.02$ ). However, results were highly heterogeneous and ranged from negatively significant, to nonsignificant, and positively significant (minimum $r=-.19$, maximum $r=.45$ ). This pattern for affective OC corresponds to existing meta-analytical evidence (Lee et al., 2000), which has pointed to a nonsignificant ( $r=.01$, based on a $k$ of 11$)$ and highly heterogeneous marriage-affective OC relationship (i.e., $41.27 \%$ of the variance between effect sizes were accounted for by artifacts). Regarding normative OC, one article found a nonsignificant correlation of $r=.01$ (Cunningham et al., 2012), whereas another found a positive correlation of $r=.11$ (Yuan and Li, 2012). For continuance OC, the only identified correlation was $r=.12$ (Cunningham et al., 2012). Worth mentioning, Wallace (1997)—who already disparaged that studies on side-bet factors, such as marriage, fail to measure continuance $\mathrm{OC}$ - found no association between marriage and continuance $\mathrm{OC}$ within a multiple regression analysis.

In sum, for affective OC, the correlation patterns suggest a relatively complex relationship with marriage, where moderators might play a role. For instance, one study found that being married was positively associated with affective OC for men, but not for women (Kaldenberg et al., 1995). 
However, past research did not provide enough results to allow for conclusions about the nature of the relationship between marriage and normative and continuance OC.

\section{Results: Life Role as Parent and Occupational Commitment}

Regarding the life role as parent, we identified only a few articles that reported correlations between either parenthood, number of dependents, or age of the youngest child and affective OC. On the one hand, positive relationships between having young children (Kaldenberg et al., 1995), age of youngest child (Cherniss, 1991), and parenthood per se (Cohen, 1999), and affective OC, were found. On the other hand, there were also some articles that identified a nonsignificant (e.g., Lee and Steele, 2009, Zhan et al., 2013, Buse and Bilimoria, 2014) or negative (Steffy and Jones, 1988) relationship between the of number of dependents and affective OC. One article also showed that the number of dependents was unrelated to continuance OC (Wallace, 1997). Regarding moderating influences, for those women with fewer children, affective OC decreased with age. For women with more children, affective OC increased with age (Buse and Bilimoria, 2014). In sum, these results suggest that adults with young children are slightly more affectively committed to their occupation, whereas the number of dependents seems to be unrelated to affective OC. For normative and continuance OC, we are unable to draw conclusions due to the limited number of studies.

\section{Results: Occupational Roles and Occupational Commitment}

We identified some articles that provided information about the relationship between holding specific occupational roles (mostly being in a higher organizational hierarchy position) and OC. Out of seven cases, five cases showed that being in a position with higher rank (e.g., supervisor, senior partner, top management) was associated with higher levels of a person's emotional attachment to their occupation (e.g., Gazica and Spector, 2015, Morrow and Wirth, 1989). The two other cases found nonsignificant effects (e.g., Weng and Mcelroy, 2012), hence, no negative relationships of hierarchical rank and affective OC have been found. Other articles have found that professional employees and union members are more emotionally attached to their occupation than are nonprofessionals and non-union members (Kim and Mueller, 2011), or that having an nonexempt status is positively related to affective OC (Goulet and Singh, 2002). We also identified three articles that 
examined links between specific features of occupational roles (e.g., role ambiguity or conflict) and OC (Blau et al., 1993, Mueller et al., 1999, Kim et al., 2013). For example, using a longitudinal design, Blau et al. (1993) found a negative effect of role ambiguity on affective OC in a sample of U.S. nurses. Further, Kim et al. (2013) examined the influence role clarity on OC in a sample of employees from a South Korean governmental energy provider. They found that role clarity was moderately positively associated with OC. The third study showed that affective OC of teachers was not influenced by role conflict (Mueller et al., 1999).

In sum, these results suggest that higher rank, degree of professionalization of one's job, unionization, and role clarity are associated with higher emotional attachment to one's occupation. However, due to the absence of studies, we cannot draw conclusions about the link between occupational role characteristics and normative and continuance OC.

\section{Results: Valuing Work and Other Life Domains and Occupational Commitment}

We identified eight articles that provided information about valuing the work and/or nonwork domain. Most studies found a positive relationship between the importance individuals attached to work and affective OC (e.g., work role salience, Aryee and Tan, 1992, value of work, Blau et al., 1993, extrinsic career goals, Spurk et al., 2016). Other studies investigated the importance of aspects of the nonwork domain (e.g., Cohen, 1995, Goulet and Singh, 2002). On the one hand, Cohen (1995) showed that the total effort and importance of outside work ties (e.g., importance of political organizations or family) were positively related to affective OC. Similarly, Schmidt and Lee (2008) found a positive relationship between parental role reward value and marital role commitment and affective OC. On the other hand, in the same study, the relationship between commitment to leisure activities and affective OC was negative, and Goulet and Singh (2002) found nonsignificant effects between family involvement and affective OC. Only two articles investigated the relationship between values and different types of OC (Van Vuuren et al., 2007, Liu and Cohen, 2010). The results showed that work values are differentially related to affective, normative, and continuance OC. For example, valuing security was only positively related to affective OC, whereas valuing selfdirection was negatively related to affective and continuance OC (Liu and Cohen, 2010). 
Taken together, it seems that individuals who value the nonwork domain can use these involvements to develop an affective bond toward their occupation. However, this effect was not as consistently positive compared to valuing (specific aspects of) the work domain.

\section{Results: Interactions Between Work and Other Life Roles and Occupational Commitment}

We identified some articles that provided information about the relationship of interactions between different life roles and affective OC. On the one hand, articles showed clear negative relationships between work-nonwork conflict and affective OC (Armstrong et al., 2015, Okurame, 2012). Thereby, Okurame (2012) showed that mentoring buffers this negative relationships. On the other hand, family supportive activities (i.e., spouse support and household coping mechanism, Aryee and Tan, 1992), and work-family (Russo and Buonocore, 2012) or family-work enrichment (Russo and Buonocore, 2012), were positively related to affective OC. In sum, these results suggest that conflicts and enrichment between occupational and other life roles influence affective OC in negative and positive ways, respectively. Unfortunately, no study investigated relationships between interactions of life roles and normative and/or continuance OC.

\section{Summary}

In sum, regarding Research Question 4, the results indicate that past research has investigated a diverse set of variables related to occupational and other life roles. Overall, the results suggest that having young children, holding high-rank or professional job positions, having or developing clear occupational roles, being involved or valuing work and nonwork life aspects, and not being involved in work-family conflicts is beneficial for individuals' emotional attachment to their occupation. Married versus unmarried individuals, on average, do not differ in affective OC. Regarding potential shortcomings, most results primarily have referred to affective OC, with a lack of studies that have focused on normative and continuance OC. Furthermore, most studies have investigated the dichotomous state of holding versus not holding specific roles, but did not analyze potential explanatory mechanisms, such as which of one's own goals and expectations of others are related to such specific occupational roles.

\section{Occupational and Other Life Events as Outcomes of Occupational Commitment}


OC is not only affected by occupational and other life events, but OC can also affect the extent to which individuals experience such events. OC is a relevant antecedent for these events because it can be seen as a mind-set that binds an individual to a course of action (as outcome), which is relevant for a specific target (Meyer and Herscovitch, 2001). In that sense, turnover can be interpreted as an occupational event that is interrelated with other life decisions (e.g., family home movement), and therefore possesses high relevance within a life span framework. In addition, occupational events, such as transitioning into retirement, might also be outcomes of OC (Wang and Shultz, 2010). More generally, event-related intentions (e.g., turnover intentions) that increase the probability of actual events (Cohen, 2000), are of specific relevance in this review. Finally, it is also plausible that all three OC dimensions are related to specific events as outcomes because all dimensions possess a behavioral intention component.

Research Question 5: Which occupational and other life events and related intentions are result of affective, normative, and continuance OC?

\section{Results: Overview}

We identified 68 cases that examined the link between turnover or turnover intentions and OC. The vast majority of these cases investigated occupational $(k=33)$ and organizational $(k=39)$ turnover intentions as an outcome. Actual organizational $(k=11)$ turnover was measured less frequently, whereas actual occupational turnover was only measured as one reason of overall job turnover (Blau, 1989, Blau, 2000). Another small cluster of articles $(k=5)$ investigated the occupational event of retirement, including outcomes such as retirement intentions or bridge employment. Occasionally, single individual articles investigated specific work-related intentions that might lead to certain events as outcomes, for instance, affective OC was related to higher levels of intentions to enter the sport management profession (Cunningham et al., 2005). However, we did not identify any study that investigated non-occupational events or related intentions as outcomes of OC.

\section{Results: Occupational Commitment and Turnover}

The correlation pattern of articles on occupational and organizational turnover intentions supports meta-analytical findings (Lee et al., 2000). Going beyond the meta-analysis, we identified 
several correlations related to normative and continuance OC. Nine studies found consistently negative correlations between normative $\mathrm{OC}$ and occupational and organizational turnover intentions (seven in case of continuance OC), which closely mirrors the results on affective OC. The correlations between OC and actual organizational turnover were also negative, but smaller than the one's between OC and organizational turnover intentions. Unfortunately, we cannot report clear findings about actual occupational turnover as an outcome of OC because actual occupational turnover was only measured as one reason of overall job turnover. However, when occupational turnover was one part of the outcome variable (e.g., Blau, 1989, Blau, 2000), the results were negative, as expected. In sum, these differentiated results on OC dimensions contribute to research on the OC-turnover link because prior reviews primarily focused on affective OC. The results suggest that (intentions of) changing or leaving one's organization or occupation do not differ between affective, normative, and continuance OC.

Some articles have gone beyond the examination of main effects and also analyzed mediators and moderators for the OC-turnover link. Regarding mediation processes, one article (Blau, 1989) found that occupational turnover intentions mediated the relation between affective OC and organizational turnover. Another article (Stinglhamber et al., 2002) found that affective organizational commitment mediated the negative relationship between affective and normative OC and organizational turnover. In another article (Cohen, 2000), the authors concluded that only turnover intentions, but not group commitment and job involvement, mediate the affective OCorganizational turnover link. In sum, these results suggest that the affective OC-actual organizational turnover link can be explained by organizational commitment and different turnover intentions.

Regarding moderation effects, some articles analyzed whether different commitment foci or commitment dimensions moderate the effects of each other. For instance, one article (Blau and Holladay, 2006) found that continuance OC moderated the link between normative OC and turnover intentions, such that this relation became more negative when continuance OC was low (vs. high). In contrast, another article (Jones and Mcintosh, 2010) found weak support for interaction effects among the components of OC when predicting organizational turnover. The article by Kim and Chang (2014) 
revealed that the relationship between organizational commitment and organizational turnover intentions was more negative when affective OC was high. Finally, one article showed that sector affiliation (high tech versus low tech IT workers) moderated the relationship between affective OC and intentions to leave the organization (Koslowsky et al., 2012). The relation was only negative in the low-tech group, whereas workers with a high OC within the high-tech sector showed the highest turnover intentions. In sum, these results suggest that other commitment foci and dimensions, as well as sector type, should be considered as moderators in the OC-turnover relationship.

\section{Results: Occupational Commitment and Retirement}

On the one hand, researchers showed that affective $\mathrm{OC}$ was a negative predictor of retirement intentions (Schmidt and Lee, 2008), and a positive predictor of planned retirement age (Adams, 1999). This suggests that individuals who are emotionally attached to their occupation intend to retire later. On the other hand, two articles (Blau, 2000, Jones and Mcintosh, 2010) found no relationship between affective OC and retirement intentions. Blau (2000) also assumed that OC and retirement intentions should be unrelated because the decision to retire would transcend the immediate occupational context and should be dependent on financial considerations as well. The article by Jones and Mcintosh (2010) analyzed effects of different kinds of commitment on bridge employment (i.e., any paid work after an individual retires or starts receiving a pension). They found that affective OC predicted bridge employment within the same occupation (here: pharmacists) rather than bridge employment in a different occupation. A similar effect of affective OC on bridge employment was found in the study by Zhan et al. (2013), which was based on a sample of Chinese retirees of medical research institutes and a postsecondary educational institution. Regarding potential moderation effects, the latter study showed that the effect of affective OC on bridge employment decisions was stronger under conditions of low economic stress, presumably because the bridge employment decision is more likely to reflect attitudinal preferences under such low-stress conditions. The article by Adams (1999) tested, but did not find interactions between age, career growth opportunities, and occupational goal attainment on planned retirement age.

\section{Summary}


Taken together, regarding Research Question 5, the review showed that research on events as outcome of OC primarily focused on different kinds of turnover and turnover-related intentions, including some studies on transitions into retirement. Overall, we can conclude that individuals who are less committed to their occupation show higher levels of intentions to leave the occupation or organization. In addition, findings from moderation analyses suggest that the link between OC and turnover is affected by more complex interaction patterns with other commitment foci (e.g., the joint impact of continuance and normative OC is specifically important for developing turnover intentions). A clear shortcoming in this research field is a lack of articles that measure actual occupational turnover, or investigate moderators for the OC-actual occupational turnover link. Regarding articles on retirement decisions as an outcome, the review suggests that individuals who are emotionally attached to their occupations intend to retire later and are also willing to continue working in the same occupation after retirement. Studies which differentiate OC into its affective, normative, and continuance components are missing, to date.

\section{Development of Occupational Commitment over Time}

As the life span perspective describes the evolution of individuals over the course of their lives, and thereby implies OC developments over time, it is of central importance to understand the stability or malleability of the OC construct in this systematic review. Past research already acknowledged that commitment should be malleable due to an individuals' processing of internal and external states, which are considered to develop and change across one's life span (Klein et al., 2012). Furthermore, the previously mentioned new career theories (Sullivan and Baruch, 2009, Hall et al., 2018) suggest that OC might currently not be very stable because a kind of malleability and flexibility is useful for navigating labor markets nowadays, and because impactful life and career events (shocks) occur more frequently compared to decades ago (Akkermans et al., 2018).

Although some of the above reviewed results of life span concepts implicitly address development over time (e.g., effects of age or career stages, or experienced specific events), and although we already referred to longitudinal studies, most studies were unable to track the development of OC over time due to methodological aspects. For instance, a cross-sectional 
relationship between age and OC not only includes information about time, but also about cohort effects and other confounding influences (Singer and Willet, 2003). Referring back to the integrative organizing framework in Figure 1, the feedback loops from occupational and other life events as outcomes, back to OC changes and other predictor categories, symbolize the dynamic developmental aspect that is, at least implicitly, covered by most life span perspectives. One important precondition to studying OC developments is a certain degree of malleability, which allows one to analyze the effect on, or of, OC changes. From a methodological point of view, temporal or longitudinal construct stability or malleability refers to the lack of change, or existence of change, in the construct over time, respectively (Bashkov and Finney, 2013). Typically, researchers apply repeated measures designs with at least two time points (ideally at least three time points) to examine rank order or mean-level consistency or change, to draw inferences about construct stability or malleability (Marsh and Grayson, 1994).

Research Question 6: How stable or malleable are affective, continuance, and normative OC on a rank order and mean level, and what do we know about drivers or outcomes of this stability or malleability?

\section{Results: Empirical Insights About Stability and Malleability of Occupational Commitment}

We identified 20 cases that measured OC at least twice (affective OC: 20 studies, normative OC: 1 study, continuance OC: 1 study), with four cases using three time points and one case using five time points. In total, the analyzed time spans ranged between 7 weeks and 36 months, meaning that only time-restricted fragments of individual careers have been longitudinally investigated. These 20 cases reported 10 rank-order stability coefficients and 23 mean differences. Regarding rank-order stability, the lowest coefficient was $r=.47$ (12 months) for affective OC, and the highest coefficient was $r=.83$ (also 12 months) for affective OC, with an average stability of $r=.65(S D=.11)$, suggesting a moderate stability of affective OC. Regarding mean stability, the largest mean decrease was 2.00 (2 months, scale from 1 to 5 ) for affective OC, and the largest mean increase was 1.03 (6 months, scale from 1 to 5) for affective OC, with an average mean change of -.06 $(S D=.86)$, suggesting a relatively stable concept on the mean level across studies, combined with a relatively 
large variance between studies. The only article that also referred to other commitment dimensions showed a weak mean decrease (delta $M=-.07$ ) of normative OC, and no mean change (delta $M=$ - .01) for continuance OC, within a sample of student nurses across a time span of 12 months (Meyer et al., 1993).

We also tested the assumption that within more recent articles (after the year 2000), due to assumptions of new career theories, OC should be more malleable compared to earlier articles (before the year 2000). However, there were no meaningful differences between OC rank order or mean stabilities in articles conducted before and after the year 2000. Some of the reviewed studies have reported OC stability with the aim of supporting re-test reliability of the applied measurement of OC (e.g., Blau et al., 1993), whereas others directly focused on the stability of OC, both from a theoretical and empirical lens (e.g., Duffy et al., 2014, Thompson and Van De Ven, 2002, Vandenberg and Scarpello, 1994).

Focusing on the reciprocal structure of relationships, an article by Duffy et al. (2014) analyzed cross-lagged effects between affective OC and living a calling in a sample of working adults across a time span of 6 months and three measurement points. Affective OC was more stable than living a calling, and results furthermore suggested that affective $\mathrm{OC}$ is more predictive for changes in living a calling than vice versa. These findings suggest that the emotional attachment of a person to her/his occupation is slightly more stable than other work-related attitudes (i.e., calling). Another article (Thompson and Van De Ven, 2002) found that groups of physicians within an occupational change process showed either decreasing or increasing developments of affective OC commitment. Furthermore, direction of the change did not differ by the life span concept of career stage (i.e., occupational tenure). This study suggests that actual occupational change processes can longitudinally increase or decrease affective OC, but potential explanations for these findings still need to be identified. Finally, it seems that affective OC is more predictive for the change in affective organizational commitment than vice versa across a time span of five months (Vandenberg and Scarpello, 1994).

\section{Summary}


In sum, regarding Research Question 6, especially after considering the rank-order and meanstability and malleability pattern, the review of the literature of longitudinal studies with multiple OC measurements suggests that $\mathrm{OC}$ is a concept that has stable components and possesses enough malleability as a precondition to investigating predictors and outcomes of this OC change from a life span perspective. However, due to the scarcity of studies that directly investigated antecedents of or outcomes, of such changes, we only have a rudimentary understanding of nomological net of OC changes. A further shortcoming is that past research did not clearly disentangle within- and betweenperson change processes, and thereby failed to provide a comprehensive account of OC change over a life span.

\section{Empirical Studies that Integrated Different Life Span Concepts}

Up to now, we have primarily focused on review results that can be clearly linked to single life span concepts (introduced above) and associated research questions. However, the review framework presented in Figure 1 also refers to more complex integrative models that might account for interdependencies between different life span concepts. For example, a study that investigates a mediating effect from career stages to OC via occupational and other life events or roles would be highly relevant from a life span perspective. Studies that adapted such rationales found, for example, that career stages, in terms of tenure, impact affective OC through valuing leisure (Cunningham et al., 2012, Tang et al., 2012), that an increase in affective OC across career stages might be due to an increase in security needs across career stages (Adler and Aranya, 1984), that work-nonwork role enrichment increases OC, which, in turn, leads to higher occupational turnover intentions (Russo and Buonocore, 2012), that different life span concepts predict one's own variance portions in affective OC (Okurame, 2012), or that age relates to affective OC via work engagement, and moderated the effects of the life role of being a parent (Buse and Bilimoria, 2014). Although some studies drew conclusions about multiple life span concept effects and OC, past research primarily investigated the life span concepts (from Figure 1) independently from each other, or treated central life span concepts (e.g., age or marriage) as control variables.

\section{Overall Summary and Contribution}


Up to now, we conducted a systematic review of the quantitative literature on OC from a life span perspective. Overall, results of our systematic literature review provide a comprehensive overview of the existing literature on the relationship between life span concepts and affective, normative, and continuance OC. Specifically, we identified several results that can be subsumed under a priori defined life span concepts that we linked to specific research questions within the organizing and integrating framework in Figure 1: age, career stages, occupational and other life roles, and occupational and other life events.

Thereby, and by differentiating results by OC types, we contributed to past research on OC in several ways. Overall, we showed that age is more strongly positively related to continuance OC than to affective and normative $\mathrm{OC}$, and that nonlinear and moderating influences are relevant in the case of the latter types (Research Question 1 in Figure 1). The same implications, but even more pronounced, can be drawn from the results about career stages in terms of occupational and organizational tenure: positive relationships with continuance OC, but more complex and presumably nonlinear relationships with affective OC (Research Question 2 in Figure 1). These findings expand past (review) work that largely focused on the relationships between age and tenure and affective OC (Lee et al., 2000, Wallace, 1997). Next, work-related transitions, on-the-job events, and macro-events affect OC in diverse ways. Generally, positively experienced or anticipated events are positively related to OC, whereas negatively experienced or anticipated events are negatively related to OC. Large scale, commonly shared macro events might even increase affective OC (Research Question 3 in Figure 1). These results support the life span focus, and suggest that the adaptation of resources due to life events is one important predictor for OC (Holmes and Rahe, 1967a, Baltes et al., 2014). Regarding occupational and other life roles, the results suggest that having young children, holding high-rank or professional job positions, having or developing clear occupational roles, being involved or valuing work and nonwork life aspects, and not being involved in work-family conflicts is beneficial for affective OC (Research Question 4 in Figure 1). These results provide a new look at past research that primarily focused on being married, or number of dependents (Lee et al., 2000), but did not provide a detailed investigation of different roles. Research on events as outcomes of OC was 
dominated by turnover and turnover intentions, but also included retirement issues. We expanded past review work by considering normative and continuance OC beyond affective OC, and by providing an initial overview of retirement issues as outcomes, which possible moderators might affect the respective relationships. Overall, independently from type of $O C$, individuals who are less committed to their occupation show higher levels of intentions to leave the occupation or organization, whereas low levels of more than one OC type facilitates this relationship (Research Question 5 in Figure 1). Finally, the first available review of the literature of longitudinal studies with multiple OC measurements suggests that $\mathrm{OC}$ is a concept that has stable components and possesses enough malleability as a precondition to investigate predictors and outcomes of this OC change from a life span perspective.

\section{Future Research Outlook}

\section{Future Research on Different Life Span Concepts}

Age and career stages. Regarding age and career stages (see Figure 1, number 1 and 2), studies should investigate more moderators that could help to understand the heterogeneity of the direction and the strength of the effects, specifically for affective and normative OC. Moreover, dichotomization of continuous age and tenure variables needs to be overcome in future studies for several reasons: The dichotomization of variables leads to a reduction in variance, which, in turn, results in a reduction of statistical power and a reduced likelihood of detecting meaningful effects, which can yield misleading interpretations (Cohen, 1983, Maccallum et al., 2002). Therefore, future studies need to use life span concepts, such as age and tenure, as continuous variables whenever possible. In our review, several studies reported nonsignificant relationships, which stands in contrast to predictions made by relevant theories (e.g., side-bet theory, psychological life span theories). Future research should further test whether higher-order (e.g., quadratic, cubic) effects more usefully explain the link between age and tenure and affective and normative OC. Finally, age was occasionally seen as an indicator of career stages (Bishop and Solomon, 1989, Adams, 1999). However, such an operationalization stands in conflict with new career theories that assume reexperiences of stages (Sullivan and Baruch, 2009) and the experience of more micro cycles (Hall and 
Chandler, 2005) that cannot be identified with a pure age variable. Therefore, future research should apply more fine-graded measures of career stages (e.g., experiences with specific career tasks, tenure on different content aspects of the occupation, types and number of micro-learning stages), and control for age, for a less confounded analysis of career stages.

\section{Occupational and other life events as antecedents and outcomes. Compared to}

occupational events as antecedents (see Figure 1, number 3), the analysis of other life event effects on OC was nearly nonexistent. However, research on life events identified several discrete events that deeply affect individuals' overall life development, and hence, might be interesting to include in OC research. Among others, such life events might include marriage, the death of a spouse, a divorce, personal injury, and illness (Holmes \& Rahe, 1967). To reflect the dynamic interplay of life events and OC, researchers should ideally theorize and study the changes in OC before and after such a significant life event, thereby following short and long-term trajectories to gain insights into the duration of the effects of life events on OC. Specifically, the role of OC in the nomological network of career shocks (Akkermans et al., 2018) seems specifically useful from a life span perspective because it accounts for positive (desired) and negative (undesired) events, and thereby would allow for a more systematic investigation of events and their effects on OC. From a practical perspective, because some events seldom occur and are not planned in advance, the inclusion of OC measures within longitudinal big data or household panels would be desirable.

Regarding events as outcomes of OC (see Figure 1, number 5), the majority of studies still analyzed actual organizational turnover as outcome of OC (e.g., Kidd \& Green, 2006; Jones \& McIntosh, 2010). Hence, although actual occupational turnover might be a low frequency event (e.g., Blau \& Holladay, 2006), future studies should investigate actual occupational turnover, beyond occupational turnover intentions, to derive a more detailed understanding of actual occurring events as outcomes. Future studies on other events as outcomes, such as retirement, should be intensified.

Occupational and other life roles. From the occupational and other life roles (see Figure 1, number 4) that Super (1957) introduced (e.g., leisurite, citizen, worker, spouse, homemaker, parent, and pensioner), not all were adequately considered in past OC research. Future research should 
therefore also focus more on neglected life roles, such as homemaker, citizen, or pensioner, and how the experience of multiple roles jointly affects OC, or is affected by OC. For example, it would be interesting to discover how the roles of worker and parent or spouse interact to shape workers' OC, and which conditions need to be fulfilled so that enactment of multiple roles can foster, rather than diminish, one's OC. Furthermore, most studies have investigated the dichotomous state of holding versus not holding specific roles. Future research should therefore operationalize relevant associated personal experiences or cognitions, such as personal values and goals, as well as expectations from others that are related to holding specific values and goals.

\section{General Recommendations for Future Research}

\section{Contesting competing theoretical assumptions and investigating integrative models.}

Generally, the literature on the link between life span concepts and OC lacks any contesting of different theoretical propositions. For example, although side-bet theory (Becker, 1960) predicts a positive relationship between age and (continuance) OC, psychological life span theories (e.g., Carstensen et al., 1999) assume a nonlinear and more complex relationship, based on changing goal priorities. The results of our research suggest that different theoretical backgrounds might be valid for different types of OC. Future research should aim to integrate predictions from life span and career stage theories, and theories that have typically been applied in OC research (e.g., side-bet theory or role theory), to contribute to the clarification of the nature of the relationship between life span concepts and different types of OC. For example, researchers could test these competing theoretical assumptions by including constructs that represent both theories (e.g., investments in one occupation vs. socioemotional motives) in their studies, to test which perspective explains more variance in OC (while controlling for the other explanation). Furthermore, because past research primarily investigated the life span concepts (from Figure 1) independently from each other, more individual empirical studies should test more integrative models that include mediating and moderating influences between different life span concepts and possible explanations (e.g., goals, resource adaptations, or expectations of others). 
Considering new career frameworks. Next, although highly relevant for commitment in the world of work, we identified only a few studies (e.g., Buse and Bilimoria, 2014, Kuron et al., 2016) that applied a new career perspective for the explanation of the relationship between any life span concept and occupational commitment. This was surprising because new career theories, such as protean, boundaryless, or kaleidoscope careers (Sullivan and Baruch, 2009), might also be fruitful for explaining why life or career events foster or decrease OC (Buse and Bilimoria, 2014). Specific related career attitudes, such as self-directed career management or mobility preferences (Briscoe et al., 2006), might also moderate relationships between life span concepts (e.g., age, career shocks, career turnover) and occupational commitment, and therefore have the potential to solve some discrepancies described above. Similar to research on new careers and organizational commitment (e.g., Briscoe and Finkelstein, 2009), the specifics of new careers in the context of OC should be investigated in more detail in future research. Related to this, future research should try to explain how past and future mobility transition profiles, or specific new employment relationships (e.g., gigwork), affect or are affected by different types of OC.

Investigating change and directions of causality. The trustworthiness and interpretability of the current findings is often limited due to the fact that the majority of studies that examined the relationships between life span concepts and OC used cross-sectional research designs (see Table 1). As life span concepts and theories imply development and change over time, future research needs to apply multiple OC measurements across time to uncover the temporal aspect of OC, in particular, in relation to life span concepts. Varying time frames combined with specific data analytical tools, such as latent growth curve modeling, could help to answer the following questions: Does OC linearly increase or decrease over career stages, and what is the variability of such trajectories between individuals? What are the correlates of interindividual variability in these intraindividual changes? Related to this issue, most studies framed OC either as an antecedent or an outcome of life span concepts, which implies causation. However, questions about causation can only be answered when using experimental research designs. Consequently, future studies should explore the possibility of laboratory and field experiments to specify the causal relationships between life span concepts and 
OC. For instance, OC could be developed through interventions in a field setting, and the effects on life span concepts, such as turnover and retirement intentions, could be examined while focusing on explaining mechanisms in this process. Interestingly, we did not identify any controlled intervention study that aimed to affect the OC of its participants through systematically varying group conditions. Furthermore, variables, such as age and tenure, could be manipulated in experimental vignette methodologies (Aguinis and Bradley, 2014), and their effect on OC perceptions from third party raters could be tested.

Occupational commitment as a multidimensional construct. Regarding the operationalization of OC dimensions, we observed that studies related to some research questions from Figure 1 (i.e., Research Question 3, 4, and 6) did not acknowledge and operationalize the multidimensional nature of the OC construct (see Table 1). At the same time, the review of studies showed that specific OC dimensions might have specific relations with life span concepts (e.g., Blau and Holladay, 2006), and that specific OC dimensions interact with each other to affect specific life span concepts. As another example, continuance commitment might show a different predictive validity regarding turnover across the life span compared to the other dimensions. We do not propagate that every study necessarily has to measure multi-dimensionally, as this surely also depends on the specific research question. Nonetheless, future studies should overcome this operationalization shortcoming and measure and hypothesize on a more multidimensional level from a life span perspective. Recently, organizational commitment research started to account for multidimensional nature by conducting latent profile analyses (Stanley et al., 2013). Such approaches should also be identified within OC research (Morin et al., 2015).

Cross-cultural and cross-occupational investigations. Most studies that we analyzed used samples from Western industrialized economies (e.g., Europe and the United States), and focused on white-collar and highly-skilled professionals (see Table 1). The current state of research might therefore not be generalizable to employees from different cultural backgrounds and to blue-collar workers. For example, employees in more collectivistic cultures (Hofstede, 2001), compared to Europe and the United States, might place less importance on individual career-related advancement 
compared to collective goals, which might influence the relationships between life span concepts and OC. Furthermore, blue-collar workers might attach different meaning to their work compared to white-collar workers (Hu et al., 2010), and relationships between job-related factors and life span concepts such as retirement intentions, have been shown to differ between white- and blue-collar workers (Schreurs et al., 2010). Consequently, future research needs test whether the current findings, with regard to the link between life span concepts and OC, can be replicated in more collectivistic cultures and using samples with blue-collar workers. Importantly, future research should also use these variables (e.g., culture, blue- vs. white-collar) as moderators to examine whether the nature and the strength of the relationships are dependent on the contextual characteristics of the sample.

\section{Conclusion}

This systematic and integrative review organized the OC literature from a life span perspective, thereby providing an alternative viewpoint on the topic, while considering several relevant theoretical approaches (e.g., side-bet theory, life span and career stage theories, and new career models). Our review revealed that several studies—either explicitly or implicitly—applied a life span perspective. We hope that our review stimulates future research that can further draw upon theoretical life span approaches together with methodological advancements to foster dynamic career development thinking, in order to move the research field of OC forward in the upcoming years. 


\section{References}

Adams, G.A. (1999), "Career-related variables and planned retirement age: An extension of Beehr's model", Journal of Vocational Behavior, Vol. 55 No. 2, pp. 221-235, doi: 10.1006/jvbe.1998.1679.

Adler, S. and Aranya, N. (1984), "A comparison of the work needs, attitudes, and preferences of professional accountants at different career stages", Journal of Vocational Behavior, Vol. 25 No. 1, pp. 45-57, doi: 10.1016/0001-8791(84)90035-6.

Aguinis, H. and Bradley, K.J. (2014), "Best practice recommendations for designing and implementing experimental vignette methodology studies", Organizational Research Methods, Vol. 17 No. 4, pp. 351-371, doi: 10.1177/1094428114547952.

Akkermans, J., Seibert, S.E. and Mol, S.T. (2018), "Tales of the unexpected: Integrating career shocks in the contemporary careers literature", SA Journal of Industrial Psychology, Vol. 44 No. 0, p. a1503, doi: 10.4102/sajip.v44i0.1503.

Armstrong, D.J., Brooks, N.G. and Riemenschneider, C.K. (2015), "Exhaustion from information system career experience: Implications for turn-away intention", Management Information System Quarterly, Vol. 39 No. 3, pp. 713-727, doi: 10.25300/misq/2015/39.3.10.

Arnold, J. (1990), "Predictors of career commitment: A test of three theoretical models", Journal of Vocational Behavior, Vol. 37 No. 3, pp. 285-302, doi: 10.1016/0001-8791(90)90046-5.

Arthur, M.B., Khapova, S.N. and Wilderom, C.P.M. (2005), "Career success in a boundaryless career world", Journal of Organizational Behavior, Vol. 26 No. 2, pp. 177-202, doi: 10.1002/job.290.

Aryee, S., Chay, Y.W. and Chew, J. (1994), "An investigation of the predictors and outcomes of career commitment in three career stages", Journal of Vocational Behavior, Vol. 44 No. 1, pp. 1-16.

Aryee, S. and Tan, K. (1992), "Antecedents and outcomes of career commitment", Journal of Vocational Behavior, Vol. 40 No. 3, pp. 288-305, doi: 10.1016/0001-8791(92)90052-2.

Baltes, P.B. (1987), "Theoretical propositions of life-span developmental psychology: On the dynamics between growth and decline", Developmental Psychology, Vol. 23 No. 5, pp. 611-626, doi: 10.1037/0012-1649.23.5.611. 
Baltes, P.B., Featherman, D.L. and Lerner, R.M. (eds.) 2014. Life-span development and behavior: Psychology Press.

Baruch, Y., Grimland, S. and Vigoda-Gadot, E. (2014), "Professional vitality and career success: Mediation, age and outcomes", European Management Journal, Vol. 32 No. 3, pp. 518-527, doi: 10.1016/j.emj.2013.06.004.

Bashkov, B.M. and Finney, S.J. (2013), "Applying longitudinal mean and covariance structures (LMACS) analysis to assess construct stability over two time points", Measurement and Evaluation in Counseling and Development, Vol. 46 No. 4, pp. 289-314, doi: 10.1177/0748175613497038.

Becker, H.S. (1960), "Notes on the concept of commitment", American Journal of Sociology, Vol. 66 No. 1, pp. 32-40, doi: $10.1086 / 222820$.

Biddle, B.J. (1986), "Recent developments in role theory", Annual Review of Sociology, Vol. 12 No. 1, pp. 67-92, doi: 10.1146/annurev.so.12.080186.000435.

Bishop, R.C. and Solomon, E. (1989), "Sex differences in career development: Locus of control and career commitment effects", Psychological Reports, Vol. 65 No. 1, pp. 107-114.

Blau, G. (1989), "Testing the generalizability of a career commitment measure and its impact on employee turnover", Journal of Vocational Behavior, Vol. 35 No. 1, pp. 88-103, doi: 10.5465/AMBPP.1989.4977932

Blau, G. (1999), "Early-Career Job Factors Influencing the Professional Commitment of Medical Technologists", Academy of Management Journal, Vol. 42 No. 6, pp. 687-695, doi: $10.2307 / 256989$.

Blau, G. (2000), "Job, organizational, and professional context antecedents as predictors of intent for interrole work transitions", Journal of Vocational Behavior, Vol. 56 No. 3, pp. 330-345, doi: 10.1006/jvbe.1999.1718.

Blau, G. (2003), "Testing for a four-dimensional structure of occupational commitment", Journal of Occupational and Organizational Psychology, Vol. 76 No. 4, pp. 469-488, doi: 10.1348/096317903322591596. 
Blau, G. and Holladay, B.E. (2006), "Testing the discriminant validity of a four-dimensional occupational commitment measure", Journal of Occupational and Organizational Psychology, Vol. 79 No. 4, pp. 691-704, doi: 10.1348/096317905x69591.

Blau, G., Paul, A. and St. John, N. (1993), "On developing a general index of work commitment", Journal of Vocational Behavior, Vol. 42 No. 3, pp. 298-314, doi: 10.1006/jvbe.1993.1021.

Blau, G.J. (1985), "The measurement and prediction of career commitment", Journal of Occupational Psychology, Vol. 58 No. 4, pp. 277-288, doi: 10.1111/j.2044-8325.1985.tb00201.x.

Blustein, D.L., Ellis, M.V. and Devenis, L.E. (1989), "The development and validation of a twodimensional model of the commitment to career choices process", Journal of Vocational Behavior, Vol. 35 No. 3, pp. 342-378, doi: Doi 10.1016/0001-8791(89)90034-1.

Blythe, J., Baumann, A., Zeytinoglu, I.U., Denton, M., Akhtar-Danesh, N., Davies, S. and Kolotylo, C. (2008), "Nursing generations in the contemporary workplace", Public Personnel Management, Vol. 37 No. 2, pp. 137-159, doi: 10.1177/009102600803700201.

Borgatti, S.P. and Foster, P.C. (2003), "The network paradigm in organizational research: A review and typology", Journal of Management, Vol. 29 No. 6, pp. 991-1013, doi: 10.1016/S01492063_03_00087-4

Briscoe, J.P. and Finkelstein, L.M. (2009), "The 'new career' and organizational commitment: Do boundaryless and protean attitudes make a difference?", Career Development International, Vol. 14 No. 3, pp. 242-260, doi: 10.1108/13620430910966424.

Briscoe, J.P., Hall, D.T. and Frautschy Demuth, R.L. (2006), "Protean and boundaryless careers: an empirical exploration", Journal of Vocational Behavior, Vol. 69 No. 1, pp. 30-47, doi: 10.3926/ic.309.

Brooks, L., Cornelius, A., Greenfield, E. and Joseph, R. (1995), "The relation of career-related work or internship experiences to the career development of college seniors", Journal of Vocational Behavior, Vol. 46 No. 3, pp. 332-349.

Burke, R.J., Astakhova, M.N. and Hang, H. (2015), "Work passion through the lens of culture: Harmonious work passion, obsessive work passion, and work outcomes in Russia and China", Journal of Business and Psychology, Vol. 30 No. 3, pp. 457-471. 
Buse, K.R. and Bilimoria, D. (2014), "Personal vision: Enhancing work engagement and the retention of women in the engineering profession", Frontiers in Psychology, Vol. 5, pp. 1-13, doi: 10.3389/fpsyg.2014.01400.

Carson, K.D. and Bedeian, A.G. (1994), "Career commitment: Construction of a measure and examination of its psychometric properties", Journal of Vocational Behavior, Vol. 44 No. 3, pp. 237-262.

Carstensen, L.L. (2006), "The influence of a sense of time on human development", Science, Vol. 312 No. 5782, pp. 1913-1915, doi: 10.1126/science.1127488.

Carstensen, L.L., Isaacowitz, D.M. and Charles, S.T. (1999), "Taking time seriously: A theory of socioemotional selectivity", American Psychologist, Vol. 54 No. 3, pp. 165-181, doi: 10.1037//0003-066x.54.3.165.

Chang, J.Y. and Choi, J.N. (2007), "The dynamic relation between organizational and professional commitment of highly educated research and development (R\&D) professionals", The Journal of Social Psychology, Vol. 147 No. 3, pp. 299-315, doi: 10.3200/SOCP.147.3.299-315.

Cherniss, C. (1991), "Career commitment in human service professionals: A biographical study", Human Relations, Vol. 44 No. 5, pp. 419-437, doi: 10.1177/001872679104400501.

Christensen, A.L. and Woodland, A.M. (2016), "Is participation in the volunteer income tax assistance (VITA) program associated with students' problem-solving skills and professional commitment?", Issues in Accounting Education, Vol. 31 No. 1, pp. 71-90, doi: 10.2308/iace-51197.

Cohen, A. (1995), "An examination of the relationships between work commitment and nonwork domains", Human Relations, Vol. 48 No. 3, pp. 239-263, doi: 10.1177/001872679504800302.

Cohen, A. (1999), "Relationships among five forms of commitment: An empirical assessment", Journal of Organizational Behavior: The International Journal of Industrial, Occupational and Organizational Psychology and Behavior, Vol. 20 No. 3, pp. 285-308.

Cohen, A. (2000), "The relationship between commitment forms and work outcomes: A comparison of three models", Human Relations, Vol. 53 No. 3, pp. 387-417, doi: 10.1177/0018726700533005.

Cohen, J. (1983), "The cost of dichotomization", Applied Psychological Measurement, Vol. 7 No. 3, pp. 249-253, doi: 10.1177/014662168300700301. 
Cooper-Hakim, A. and Viswesvaran, C. (2005), "The construct of work commitment: Testing an integrative framework.", Psychological Bulletin, Vol. 131 No. 2, pp. 241-259.

Cunningham, G.B., Sagas, M., Dixon, M., Kent, A. and Turner, B.A. (2005), "Anticipated Career Satisfaction, Affective Occupational Commitment, and Intentions to Enter the Sport Management Profession", Journal of Sport Management, Vol. 19 No. 1, pp. 43-57, doi: 10.1123/jsm.19.1.43.

Cunningham, P.H., Tang, T.L.-P., Frauman, E., Ivy, M.I. and Perry, T.L. (2012), "Leisure Ethic, Money Ethic, and Occupational Commitment among Recreation and Park Professionals: Does Gender Make a Difference?", Public Personnel Management, Vol. 41 No. 3, pp. 421-448, doi: 10.1177/009102601204100303.

De Vos, A., Forrier, A., Van Der Heijden, B. and De Cuyper, N. (2017), "Keep the expert! Occupational expertise, perceived employability and job search", Career Development International, Vol. 22 No. 3, pp. 318-332, doi: 10.1108/CDI-12-2016-0229.

Duffy, R.D., Allan, B.A., Autin, K.L. and Douglass, R.P. (2014), "Living a calling and work well-being: A longitudinal study", Journal of Counseling Psychology, Vol. 61 No. 4, pp. 605-15, doi: 10.1037/cou0000042.

Erikson, E.H. (1963), Childhood and society, W. W. Norton \& Company.

Gazica, M.W. and Spector, P.E. (2015), "A comparison of individuals with unanswered callings to those with no calling at all", Journal of Vocational Behavior, Vol. 91, pp. 1-10.

Gould, S. (1979), "Characteristics of career planners in upwardly mobile occupations", Academy of Management Journal, Vol. 22 No. 3, pp. 539-550, doi: 10.2307/255743.

Goulet, L.R. and Singh, P. (2002), "Career Commitment: A Reexamination and an Extension", Journal of Vocational Behavior, Vol. 61 No. 1, pp. 73-91, doi: 10.1006/jvbe.2001.1844.

Greenhaus, J.H. and Kossek, E.E. (2014), "The contemporary career: A work-home perspective", Annual Review of Organizational Psychology and Organizational Behavior, Vol. 1 No. 1, pp. 361-388, doi: 10.1146/annurev-orgpsych-031413-091324.

Grover, S.L. (1992), "The effect of increasing education on individual professional behavior and commitment", Journal of Vocational Behavior, Vol. 40 No. 1, pp. 1-13.

Hall, D.T. (2002), Careers in and out of organizations, Thousand Oaks, CA, Sage. 
Hall, D.T. and Chandler, D.E. (2005), "Psychological success: When the career is a calling", Journal of Organizational Behavior, Vol. 26 No. 2, pp. 155-176, doi: 10.1002/job.301.

Hall, D.T., Yip, J. and Doiron, K. (2018), "Protean Careers at Work: Self-Direction and Values Orientation in Psychological Success", Annual Review of Organizational Psychology and Organizational Behavior, Vol. 5, pp. 129-156.

Henry, H., Zacher, H. and Desmette, D. (2015), "Reducing Age Bias and Turnover Intentions by Enhancing Intergenerational Contact Quality in the Workplace: The Role of Opportunities for Generativity and Development", Work, Aging and Retirement, Vol. 1 No. 3, pp. 243-253, doi: 10.1093/workar/wav005.

Henry, H., Zacher, H. and Desmette, D. (2017), "Future Time Perspective in the Work Context: A Systematic Review of Quantitative Studies", Frontiers in psychology, Vol. 8, pp. 1-22, doi: 10.3389/fpsyg.2017.00413.

Hertel, G. and Zacher, H. (in press), Managing the aging workforce. In: Chockalingam, V., Anderson, N., Ones, D.S. and Sinangil, H.K. (eds.) The SAGE Handbook of Industrial, Work, \& Organization Psychology. 2 ed. Thousand Oaks, CA: Sage.

Hofstede, G. (2001), Culture's consequences: Comparing values, behaviors, institutions and organizations across nations, Thousand Oaks, CA, Sage.

Holmes, T.H. and Rahe, R.H. (1967a), "The social readjustment rating scale", Journal of Psychosomatic Research, Vol. 11 No. 2, pp. 213-218.

Holmes, T.H. and Rahe, R.H. (1967b), "The social readjustment rating scale", Journal of Psychosomatic Research, Vol. 11 No. 2, pp. 213-8.

Howes, L.M. and Goodman-Delahunty, J. (2014), "Predicting Career Stability and Mobility: Embeddedness and Boundarylessness", Journal of Career Development, Vol. 42 No. 3, pp. 244259, doi: 10.1177/0894845314548722.

Hu, X., Kaplan, S. and Dalal, R.S. (2010), "An examination of blue- versus white-collar workers' conceptualizations of job satisfaction facets", Journal of Vocational Behavior, Vol. 76 No. 2, pp. 317-325, doi: 10.1016/j.jvb.2009.10.014. 
Irving, P.G., Coleman, D.F. and Cooper, C.L. (1997), "Further assessments of a three-component model of occupational commitment: Generalizability and differences across occupations", Journal of Applied Psychology, Vol. 82 No. 3, pp. 444-452, doi: 10.1037/0021-9010.82.3.444.

Jones, D.A. and Mcintosh, B.R. (2010), "Organizational and occupational commitment in relation to bridge employment and retirement intentions", Journal of Vocational Behavior, Vol. 77 No. 2, pp. 290-303, doi: 10.1016/j.jvb.2010.04.004.

Kaldenberg, D.O., Becker, B.W. and Zvonkovic, A. (1995), "Work and commitment among young professionals: A study of male and female dentists", Human Relations, Vol. 48 No. 11, pp. 13551377, doi: 10.1177/001872679504801106.

Kim, S.-W. and Mueller, C.W. (2011), "Occupational and organizational commitment in different occupational contexts: The case of South Korea", Work and occupations, Vol. 38 No. 1, pp. 3-36.

Kim, S., Egan, T.M., Kim, W. and Kim, J. (2013), "The Impact of Managerial Coaching Behavior on Employee Work-Related Reactions", Journal of Business and Psychology, Vol. 28 No. 3, pp. 315330, doi: 10.1007/s10869-013-9286-9.

Kim, T. and Chang, K. (2014), "Turnover intentions and organizational citizenship behaviours in Korean firms: The interactional effects of organizational and occupational commitment", Asia Pacific Business Review, Vol. 20 No. 1, pp. 59-77, doi: 10.1080/13602381.2011.640538.

Klassen, R.M. and Chiu, M.M. (2011), "The occupational commitment and intention to quit of practicing and pre-service teachers: Influence of self-efficacy, job stress, and teaching context", Contemporary Educational Psychology, Vol. 36 No. 2, pp. 114-129, doi: 10.1016/j.cedpsych.2011.01.002.

Klein, H.J., Molloy, J.C. and Brinsfield, C.T. (2012), "Reconceptualizing workplace commitment to redress a stretched construct: Revisiting assumptions and removing confounds", Academy of Management Review, Vol. 37 No. 1, pp. 130-151, doi: 10.5465/amr.2010.0018.

Kooij, D., Peeters, M., De Lange, A., Jansen, P. and Dikkers, J. (2008), "Older workers' motivation to continue to work: Five meanings of age", Journal of Managerial Psychology, Vol. 23 No. 4, pp. 364-394, doi: 10.1108/02683940810869015. 
Koslowsky, M., Weisberg, J., Yaniv, E. and Zaitman-Speiser, I. (2012), "Ease of movement and sector affiliation as moderators of the organizational and career commitment Turnover intentions link", International Journal of Manpower, Vol. 33 No. 7, pp. 822-839, doi: $10.1108 / 01437721211268348$.

Kuron, L.K.J., Schweitzer, L., Lyons, S. and Ng, E.S.W. (2016), "Career profiles in the "new career”: Evidence of their prevalence and correlates", Career Development International, Vol. 21 No. 4, pp. 355-377, doi: 10.1108/cdi-05-2015-0066.

Lee, G.J. and Steele, C.L. (2009), "Boundary management of employees' non-work lives: Effects on South African workers' commitment", South African Journal of Economic and Management Sciences, Vol. 12 No. 1, pp. 63-80.

Lee, K., Carswell, J. and Allen, N.J. (2000), "A meta-analytic review of occupational commitment: Relations with person and work-related variables", Journal of Applied Psychology, Vol. 85 No. 5, pp. 799-811.

Lent, R.W. and Brown, S.D. (2013), "Social cognitive model of career self-management: Toward a unifying view of adaptive career behavior across the life span", Journal of Counseling Psychology, Vol. 60 No. 4, pp. 557-68, doi: 10.1037/a0033446.

Lerner, R.M. and Busch-Rossnagel, N.A. (eds.) 2013. Individuals as producers of their development: A life-span perspective: Elsevier.

Liu, Y. and Cohen, A. (2010), "Values, commitment, and OCB among Chinese employees", International Journal of Intercultural Relations, Vol. 34 No. 5, pp. 493-506.

Low, C.H., Bordia, P. and Bordia, S. (2016), "What do employees want and why? An exploration of employees' preferred psychological contract elements across career stages", Human Relations, Vol. 69 No. 7, pp. 1457-1481, doi: 10.1177/0018726715616468.

Maccallum, R.C., Zhang, S., Preacher, K.J. and Rucker, D.D. (2002), "On the practice of dichotomization of quantitative variables", Psychological Methods, Vol. 7 No. 1, pp. 19-40, doi: 10.1037//1082989x.7.1.19.

Mainiero, L.A. and Sullivan, S.E. (2005), "Kaleidoscope careers: An alternate explanation for the "optout" revolution", The Academy of Management Executive, Vol. 19 No. 1, pp. 106-123. 
Marsh, H.W. and Grayson, D. (1994), "Longitudinal stability of latent means and individual differences: A unified approach", Structural Equation Modeling: A Multidisciplinary Journal, Vol. 1 No. 4, pp. 317-359, doi: 10.1080/10705519409539984.

Meyer, J.P., Allen, N.J. and Smith, C.A. (1993), "Commitment to organizations and occupations: Extension and test of a three-component conceptualization", Journal of Applied Psychology, Vol. 78 No. 4, pp. 538-551, doi: 10.1037/0021-9010.78.4.538.

Meyer, J.P. and Herscovitch, L. (2001), "Commitment in the workplace. Towards a general model. ", Human Resource Management Review, Vol. 11 No. 3, pp. 299-326, doi: 10.1016/S10534822(00)00053-X.

Meyer, J.P., Jackson, T.A. and Maltin, E.R. (2008), Commitment in the workplace: Past, present, and future. In: Barling, J. and Cooper, C.L. (eds.) The SAGE Handbook of Organizational Behavior: Volume I - Micro Approaches London, UK: Sage.

Mihelič, K.K. (2014), "Commitment to life roles and work-family conflict among managers in a postsocialist country", Career development international, Vol. 19 No. 2, pp. 204-221.

Morin, A.J., Meyer, J.P., Mcinerney, D.M., Marsh, H.W. and Ganotice, F.A. (2015), "Profiles of dual commitment to the occupation and organization: Relations to well-being and turnover intentions", Asia Pacific Journal of Management, Vol. 32 No. 3, pp. 717-744.

Morrow, P.C. and Wirth, R.E. (1989), "Work commitment among salaried professionals", Journal of Vocational Behavior, Vol. 34 No. 1, pp. 40-56, doi: 10.1016/0001-8791(89)90063-8.

Mount, M.K. (1984), "Managerial career stage and facets of job satisfaction", Journal of Vocational Behavior, Vol. 24 No. 3, pp. 340-354, doi: 10.1016/0001-8791(84)90017-4.

Mueller, C.W., Finley, A., Iverson, R.D. and Price, J.L. (1999), "The effects of group racial composition on job satisfaction, organizational commitment, and career commitment: The case of teachers", Work and Occupations, Vol. 26 No. 2, pp. 187-219.

Ng, T.W.H. and Feldman, D.C. (2007), "Organizational embeddedness and occupational embeddedness across career stages", Journal of Vocational Behavior, Vol. 70 No. 2, pp. 336-351, doi: 10.1016/j.jvb.2006.10.002. 
Ok, A.B. and Vandenberghe, C. (2016), "Organizational and career-oriented commitment and employee development behaviors", Journal of Managerial Psychology, Vol. 31 No. 5, pp. 930-945, doi: 10.1108/Jmp-04-2015-0157.

Okurame, D.E. (2012), "Linking work-family conflict to career commitment: The moderating effects of gender and mentoring among Nigerian Civil Servants", Journal of Career Development, Vol. 39 No. 5, pp. 423-442.

Osinsky, P. and Mueller, C.W. (2004), "Professional commitment of Russian provincial specialists", Work and Occupations, Vol. 31 No. 2, pp. 193-224.

Otto, K., Dette-Hagenmeyer, D.E. and Dalbert, C. (2010), "Occupational Mobility in Members of the Labor Force: Explaining the Willingness to Change Occupations", Journal of Career Development, Vol. 36 No. 3, pp. 262-288, doi: 10.1177/0894845309345842.

Reilly, N.P. and Orsak, C.L. (1991), "A career stage analysis of career and organizational commitment in nursing", Journal of Vocational Behavior, Vol. 39 No. 3, pp. 311-330, doi: 10.1016/00018791(91)90041-j.

Russo, M. and Buonocore, F. (2012), "The relationship between work-family enrichment and nurse turnover", Journal of Managerial Psychology, Vol. 27 No. 3, pp. 216-236, doi: $10.1108 / 02683941211205790$.

Schmidt, J.A. and Lee, K. (2008), "Voluntary retirement and organizational turnover intentions: The differential associations with work and non-work commitment constructs", Journal of Business and Psychology, Vol. 22 No. 4, pp. 297-309, doi: 10.1007/s10869-008-9068-y.

Schreurs, B., Van Emmerik, H., De Cuyper, N., Notelaers, G. and De Witte, H. (2010), "Job demandsresources and early retirement intention: Differences between blue-and white-collar workers", Economic and Industrial Democracy, Vol. 32 No. 1, pp. 47-68, doi: 10.1177/0143831x10365931.

Singer, J.D. and Willet, J.B. (2003), Applied longitudinal data analysis: Modeling change and event occurrence, New York, Oxford University Press.

Snape, E., Lo, C.W.-H. and Redman, T. (2008), "The Three-Component Model of Occupational Commitment: A Comparative Study of Chinese and British Accountants", Journal of CrossCultural Psychology, Vol. 39 No. 6, pp. 765-781, doi: 10.1177/0022022108323789. 
Spurk, D., Hirschi, A. and Kauffeld, S. (2016), "A new perspective on the etiology of workaholism. The role of personal and contextual career-related antecedents", Journal of Career Assessment, Vol. 24 No. 4, pp. 747-764.

Stanley, L., Vandenberghe, C., Vandenberg, R. and Bentein, K. (2013), "Commitment profiles and employee turnover", Journal of Vocational Behavior, Vol. 82 No. 3, pp. 176-187.

Steffy, B.D. and Jones, J.W. (1988), "The impact of family and career planning variables on the organizational, career, and community commitment of professional women", Journal of Vocational Behavior, Vol. 32 No. 2, pp. 196-212, doi: 10.1016/0001-8791(88)90014-0.

Stinglhamber, F., Bentein, K. and Vandenberghe, C. (2002), "Extension of the three-component model of commitment to five foci: Development of measures and substantive test", European Journal of Psychological Assessment, Vol. 18 No. 2, pp. 123-138, doi: 10.1027//1015-5759.18.2.123.

Stumpf, S.A. and Rabinowitz, S. (1981), "Career Stage as a Moderator of Performance Relationships with Facets of Job-Satisfaction and Role Perceptions", Journal of Vocational Behavior, Vol. 18 No. 2, pp. 202-218, doi: Doi 10.1016/0001-8791(81)90008-7.

Sullivan, S.E. and Baruch, Y. (2009), "Advances in career theory and research: A critical review and agenda for future exploration", Journal of Management, Vol. 35 No. 6, pp. 1542-1571, doi: $10.1177 / 0149206309350082$

Super, D.E. (1953), "A theory of vocational development", American Psychologist, Vol. 8 No. 5, pp. 185190, doi: 10.1037/h0056046.

Super, D.E. (1980), "A life-span, life-space approach to career development", Journal of Vocational Behavior, Vol. 16 No. 3, pp. 282-298, doi: 10.1016/0001-8791(80)90056-1.

Super, D.E., Brown, D. and Brooks, L. (1990), A life-span, life-space approach to career development. Career choice and development: Applying contemporary theories to practice (2nd ed.). JosseyBass.

Tajfel, H. (1981), Human Groups and Social Categories, Cambridge, Cambridge University Press.

Tang, T.L.-P., Cunningham, P.H., Frauman, E., Ivy, M.I. and Perry, T.L. (2012), "Attitudes and Occupational Commitment among Public Personnel: Differences between Baby Boomers and 
Gen-Xers", Public Personnel Management, Vol. 41 No. 2, pp. 327-360, doi:

10.1177/009102601204100206.

Tempest, S. and Coupland, C. (2017), "Lost in time and space: temporal and spatial challenges facing older workers in a global economy from a career capital perspective", The International Journal of Human Resource Management Vol. 28 No. 15, pp. 2159-2183, doi: 10.1080/09585192.2015.1128455.

Thompson, J.A. and Van De Ven, A.H. (2002), "Commitment shift during organizational upheaval: Physicians' transitions from private practitioner to employee", Journal of Vocational Behavior, Vol. 60 No. 3, pp. 382-404, doi: 10.1006/jvbe.2001.1819.

Ugboro, I.O. and Obeng, K. (2015), "The moderating effects of perceived threat to valued job features on career commitment among university professors", Australian Journal of Career Development, Vol. 24 No. 1, pp. 39-52, doi: 10.1177/1038416214564885.

Van Vuuren, M., Veldkamp, B.P., De Jong, M.D. and Seydel, E.R. (2007), "Why work? Aligning foci and dimensions of commitment along the axes of the competing values framework", Personnel Review, Vol. 37 No. 1, pp. 47-65.

Vandenberg, R.J. and Scarpello, V. (1994), "A longitudinal assessment of the determinant relationship between employee commitments to the occupation and the organization", Journal of Organizational Behavior, Vol. 15 No. 6, pp. 535-547.

Wallace, J.E. (1997), "Becker's side-bet theory of commitment revisited: Is it time for a moratorium or a resurrection?", Human Relations, Vol. 50 No. 6, pp. 727-749, doi: 10.1023/a:1016956322744.

Wang, J., Leung, K. and Zhou, F. (2014), "A dispositional approach to psychological climate: Relationships between interpersonal harmony motives and psychological climate for communication safety", Human Relations, Vol. 67 No. 4, pp. 489-515, doi: $10.1177 / 0018726713495423$.

Wang, M., Burlacu, G., Truxillo, D., James, K. and Yao, X. (2015), "Age differences in feedback reactions: The roles of employee feedback orientation on social awareness and utility", Journal of Applied Psychology, Vol. 100 No. 4, pp. 1296-1308, doi: 10.1037/a0038334. 
Wang, M. and Shultz, K.S. (2010), "Employee retirement: A review and recommendations for future investigation", Journal of Management, Vol. 36 No. 1, pp. 172-206.

Weng, Q. and Mcelroy, J.C. (2012), "Organizational career growth, affective occupational commitment and turnover intentions", Journal of Vocational Behavior, Vol. 80 No. 2, pp. 256-265, doi: 10.1016/j.jvb.2012.01.014.

Yousaf, A., Sanders, K. and Abbas, Q. (2015), "Organizational/occupational commitment and organizational/occupational turnover intentions: A happy marriage?", Personnel Review, Vol. 44 No. 4, pp. 470-491.

Yuan, L. and Li, J. (2012), "Occupational commitment and labor relations in firms: an empirical study in China", Chinese Management Studies, Vol. 6 No. 2, pp. 300-317.

Zacher, H. (2015), "Successful aging at work", Work, Aging and Retirement, Vol. 1 No. 1, pp. 4-25, doi: 10.1093/workar/wau006.

Zhan, Y., Wang, M. and Yao, X. (2013), "Domain specific effects of commitment on bridge employment decisions: The moderating role of economic stress", European Journal of Work and Organizational Psychology, Vol. 22 No. 3, pp. 362-375, doi: 10.1080/1359432x.2012.762763.

Zhou, Z.E., Yang, L.Q. and Spector, P.E. (2015), "Political skill: A proactive inhibitor of workplace aggression exposure and an active buffer of the aggression-strain relationship", Journal of Occupational Health Psychology, Vol. 20 No. 4, pp. 405-19, doi: 10.1037/a0039004. 


\section{OCCUPATIONAL COMMITMENT FROM A LIFE SPAN PERSPECTIVE}

Table 1.

Overview of Central Study Characteristics of Included Quantitative OC Research $(k=138)$.

\begin{tabular}{|c|c|c|}
\hline Study Characteristic & $k$ & Ratio $^{1)}(\%)$ \\
\hline \multicolumn{3}{|l|}{ Construct Dimension } \\
\hline Affective OC & 137 & $99 \%$ \\
\hline Normative OC & 32 & $23 \%$ \\
\hline Continuance OC & 25 & $18 \%$ \\
\hline $\begin{array}{l}\text { Combination of affective, normative, } \\
\text { and continuance OC }\end{array}$ & 23 & $17 \%$ \\
\hline \multicolumn{3}{|l|}{ Study Design } \\
\hline Cross-sectional study & 100 & $73 \%$ \\
\hline Longitudinal study & 38 & $28 \%$ \\
\hline Multiple OC measures & 20 & $15 \%$ \\
\hline \multicolumn{3}{|l|}{ Study Sample ${ }^{2)}$} \\
\hline \multicolumn{3}{|l|}{ Sample origin } \\
\hline Northern America & 72 & $52 \%$ \\
\hline Asia & 37 & $27 \%$ \\
\hline Europe & 18 & $13 \%$ \\
\hline Oceania & 3 & $2 \%$ \\
\hline Latin America ${ }^{3)}$ & 2 & $1 \%$ \\
\hline Africa & 2 & $1 \%$ \\
\hline \multicolumn{3}{|c|}{ Sample size $^{4)}(M=378 ; S D=316 ; \min =22 ; \max =2032)$} \\
\hline Small sample size & 14 & $10 \%$ \\
\hline Medium sample size & 115 & $83 \%$ \\
\hline Big sample size & 8 & $6 \%$ \\
\hline
\end{tabular}

Note. OC = Occupational Commitment; ${ }^{1)}$ Ratio (rounded) in relation to the total number within the respective column; ${ }^{2}$ Not all studies provided information about the origin of the sample;

${ }^{3)}$ including the Caribbean ${ }^{4)}$ Small sample size $(N \leq 100)$; Medium sample size $(N=[101,999])$;

Big sample size $(N \geq 1000)$; not all studies provided information about sample size. 

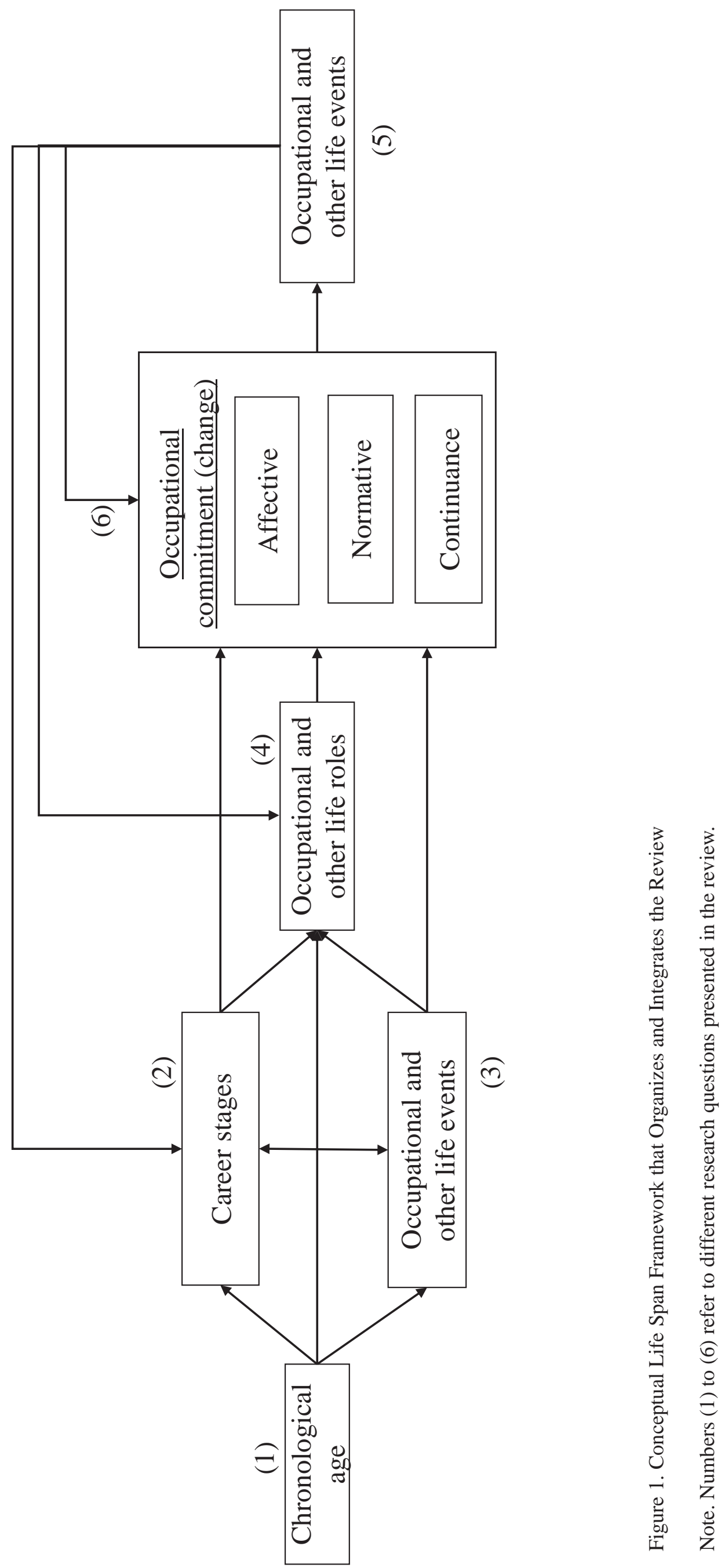


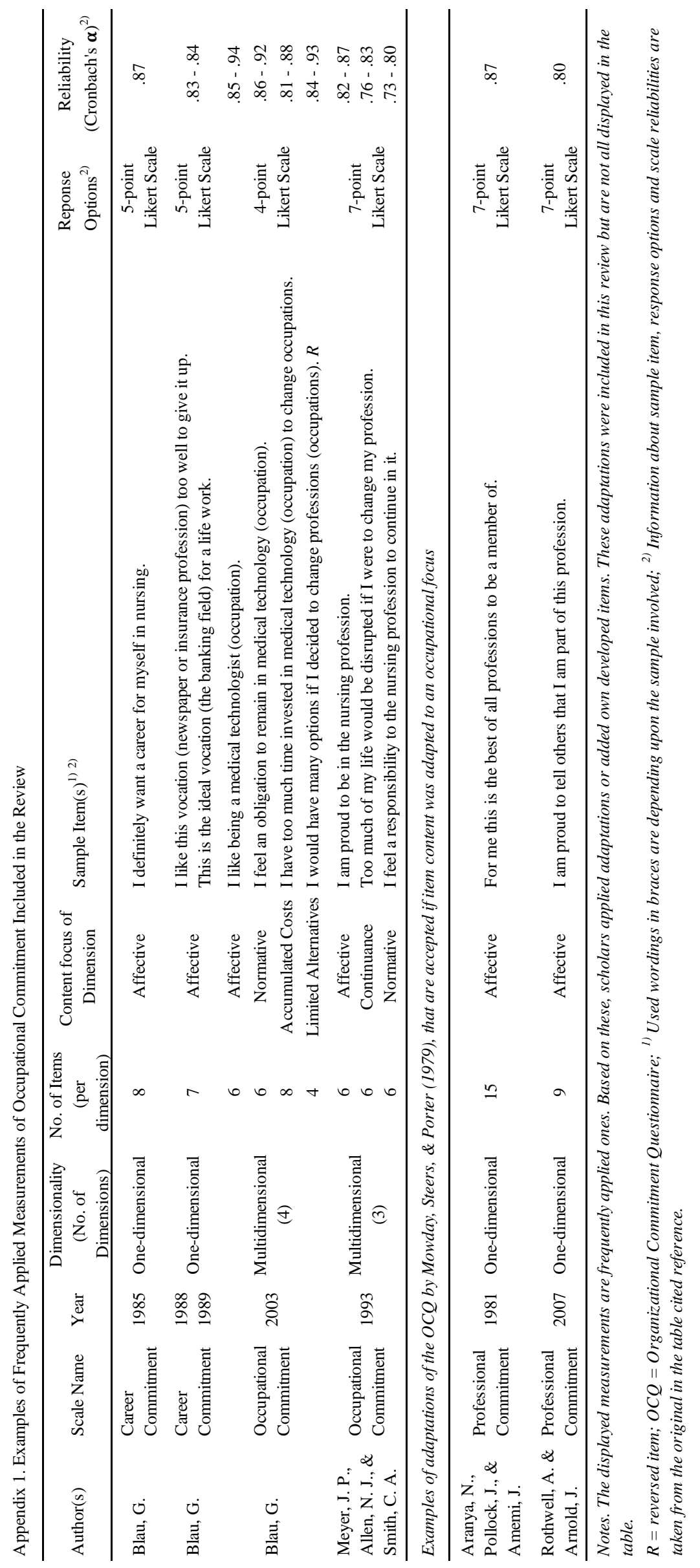

